\title{
„„... viell fürsten halten ein Musica” \\ Musik und Musiker am Hofe der Fürsten von Liechtenstein im 17. Jahrhundert
}

\section{... viell fürsten halten ein Musica... \\ Music and Musicians in the Court of the Princes of Liechtenstein in the $17^{\text {th }}$ Century}

\author{
Vladimír Maňas / manas@phil.muni.cz \\ Ústav hudební vědy, Filozofická fakulta, Masarykova univerzita, Brno, CZ
}

\begin{abstract}
The so-called Political Testament, written by Charles Eusebius the Prince of Liechtenstein for his son and heir around 1680 includes, among other things, a substantial, detailed passage dedicated to music at the prince's court. Music is not just one element of court representation here. This key aspect, in the text of the instruction, comes with a detailed insight to possible problems with a music ensemble, as well as with the use and character of individual instruments. This study focuses primarily on the status of musical ensembles and musicians within the structure of the aristocratic court, beginning with Charles I (d. 1627), through a partial analysis of the situation at the court of his son Charles Eusebius (d. 1686) and finally brings an outline of the role of musicians and musical life with the third generation of princes from Liechtenstein. Certain continuity can be assumed, in particular, in attempting to adapt its own court to princely habits (for example, as regards the minimum number of trumpeters etc). In general, however, it must be stressed that the trumpeters or the trumpeter ensemble constituted an essential part of the princely court, whereas the existence of a musical ensemble always depended on the actual needs, preferences and economic possibilities of a particular prince.
\end{abstract}

\section{Keywords}

Early Modern Age, nobility, music, Liechtenstein Princely Court, Charles I of Liechtenstein, Charles Eusebius of Liechtenstein, court band, Zangius

Das sog. Politische Testament, das Karl Eusebius Fürst von Liechtenstein zu einem nicht näher bekannten Zeitpunkt um das Jahr 1680 für seinen Sohn und Erben verfasste, 
enthält unter anderem eine grundlegende und detaillierte, der Musik am Fürstenhofe gewidmete Passage. ${ }^{1}$ Die Musik figuriert hier nicht allein nur als eines der Elemente höfischer Repräsentation. Dieser Schlüsselaspekt trifft im Text der Instruktion auf die gelehrte Sicht auf die mit dem Unterhalt eines Musikensembles und mit dem Einsatz und dem Charakter der einzelnen Instrumente verbundenen Probleme. Im Mittelpunkt dieser Studie soll jedoch vor allem die Stellung der Musikensemble und der Musiker innerhalb der Struktur des aristokratischen Hofes stehen, also eine den engen Fachbegriff sprengende Frage, die darüber hinaus mit überraschenden und bislang eher nur erahnten - den Rahmen der höfischen Repräsentation übersteigenden- Wirkungen verbunden ist. Im Rahmen dieser soziologischen Herangehensweise lassen wir Fragen individueller Vorlieben in der Musik, der eigentlichen musikalischen Aktivitäten der Aristokratie und schließlich auch des eigentlichen Musikbetriebes an den einzelnen Höfen etwas außer Acht.

\section{Die Struktur}

Für eine zumindest flüchtige Erfassung der paradigmatischen Veränderungen erweist es sich an dieser Stelle als angebracht einen breiteren zeitlichen Rahmen festzusetzen und auf die Ausgangspositionen der Musiker innerhalb der höfischen Struktur zu verweisen. Die Veränderungen, die sich seit der Mitte des 16. und vor allem im Verlaufe des 17. Jahrhunderts vollzogen, lassen sich gerade am Beispiel des Hauses Liechtenstein und seinen ersten drei Fürstengenerationen gut dokumentieren.

Einen Impuls für diesen Exkurs lieferte unter anderem die Aufforderung von Peter Mata zu einem grundlegenden Vergleich der funktionalen Struktur der Höfe. ${ }^{2}$ Im Rahmen der frühneuzeitlichen Strukturveränderungen konstatiert Mata eine Tendenz zur Demilitarisierung und Vertiefung der repräsentativen und administrativ-bürokratischen Funktion, also kurz gesagt eine Verwandlung der militärischen Gefolgschaft in reprä-

1 HAL Wien, ursprüngliche Signatur VA 5-2-2; aktuelle Signatur Hs 462 (Instruction. Von wayl: fürsten Carolo Eusebio von Liechtenstein für dero Prinzen Fürsten Johann Adam geschriben, Abschrift aus dem 18. Jahrhundert), S. 243-245. Im Zusammenhang mit dem Entwurf zur Nutzung der einzelnen Güter das Zitat bei Winkelbauer, Thomas: Fürst und Fürstendiener. Gundaker von Liechtenstein, ein österreichischer Aristokrat des konfessionellen Zeitalters. Wien - München 1999, S. 367. Wesentliche Teile der Passage über die Musik aus der gleichen Quelle sind bereits ediert, vgl. Haupt, Herbert (hg. von. Kräftner, Johann): Fürst Karl Eusebius von Liechtenstein 1611-1684. Erbe und Bewahrer in schwerer Zeit. München 2007, S. $268-269$. Die einzelnen Instruktionen Karl Eusebius' von Liechtenstein sind eingehend analysiert von Pospichal, Barbara: Zu einer richtschnuer freundtlich an die hand geben. Die Instruktion des Fürsten Karl Eusebius von Liechtenstein für die Vormünder seines Sohnes für den Fall seines Todes. Diplomarbeit Wien 2016, zur Musik v. a. S. 87-88. Im tschechischen Kontext des sog. Politischen Testaments nutzte dies in erster Linie Petra Vokáčová in ihrer Darstellung des Lebens von Erdmunda Theresia von Dietrichstein, Gemahlin des Johann Adam Andreas von Liechtenstein. Vgl. Vokáčová, Petra: Příběhy o hrdé pokoře. Aristokracie českých zemích v době baroka (Geschichten von stolzer Demut. Die Aristokratie in den böhmischen Ländern in der Barockzeit). Praha 2014, S. 340-342, 691-692.

2 Mała, Petr: Svět české aristokracie (1500-1700) [Die Welt der böhmischen Aristokratie (1500-1700)]. Praha 2004, S. 240. 
sentierende Personen. ${ }^{3}$ Der Schlüsselbegriff Musica, den in seiner Instruktion auch Karl Eusebius von Liechtenstein benutzt, und den zahlreiche neuzeitliche Quellen durchblicken lassen, hat - vielleicht auch der eigentlichen Verwendung zum Trotz - ähnliche mittelalterliche Wurzeln.

Eine kurze, freilich sehr treffende Zusammenfassung bietet Howard M. Brown: Die im Verlaufe des 15. Jahrhunderts entstandenen musikalischen Institutionen bei Hofe reflektierten die Praxis der Außenwelt. Die Mehrzahl der Herrscher beschäftigte drei Arten von Musikern. Eine ideale Norm verkörperte an dieser Stelle vor allem die Situation am Hofe des französischen Königs Franz I. (†1547), der Sänger und Organisten als musikalisches Personal seiner Kapelle (Musique de la chapelle), Instrumentalisten (Musique de l'écurie) sowie eine praktisch sehr freie Gruppe virtuoser Sänger und Instrumentalisten als individuelle Kammermusiker (Musique de chambre) beschäftigte. Die Aufgabe von Randgruppen erscheint insgesamt verständlich und gut definiert. Die mittlere Gruppe - im Falle von Franz' Hof zum einen ein Ensemble zeremonieller Trompeter und Kesselpauker, ein Ensemble von Schalmei- und Posaunenspielern und schließlich ein Ensemble von Streichinstrumenten - diente vor allem Tanz- und weiteren Galaproduktionen. ${ }^{4}$

Innerhalb der höfischen Struktur unterstanden die Musiker der Kapelle (beziehungsweise auch die Kammermusiker) dem Hofmeister, die Instrumentalisten hingegen dem Stallmeister. Dieses traditionelle Schema bezeugt beispielsweise ein Dokument aus der Zeit Kaiser Maximilians II., als in Prag 1565 die Instruction und Ordnung auf die Capeln und Instrumentisten verfasst wurde. Ungeachtet der engen Zusammenarbeit zwischen beiden Ensembles unterstanden die Sänger (Capel) dem Obersthofmeister, die Instrumentalisten hingegen dem obersten Stallmeister. Der terminologischen Unterscheidung in der Prager Instruktion entspricht ein ähnliches Duo von Termini im Falle des herzoglichen Hofes in Heidelberg für das Jahr 1558: Capell vs. Musica. ${ }^{5}$ Die erwähnte enge Zusammenarbeit beider Ensemble und die Verwischung dieser strukturellen Schemata im Rahmen der zeitgenössischen Musikpraxis unterstreichen sodann zahlreiche Fälle, in denen der Vorsteher der Instrumentalisten die Funktion des Vizekapellmeisters (stellvertretenden Leiters des Ensembles der Kapelle) bzw. direkt jene des Kapellmeisters ausübte. ${ }^{6}$

Innerhalb der etymologischen Herangehensweise erscheint es notwendig in Betracht zu ziehen, dass in späteren Zeitabschnitten der in unserer sprachlichen Gegenwart der zahlreiche anderen Bedeutungen belastete Begriff der Kapelle sich im 15. und 16. Jahrhundert ausschließlich auf das (musikalische) Personal der Hofkapelle bezog. Ursprünglich rekrutierte sich nämlich deren Personal praktisch ausnahmslos aus in ihrer Existenz durch konkrete Benefizien materiell abgesicherten Klerikern. Erst im Verlaufe des

3 Ebd., S. 241.

4 Mayer Brown, Howard - Sadie, Stanley (Hg.): Performance Practice. Music before 1600. London 1989, S. 157.

5 Reimer, Erich: Die Hofmusik in Deutschland 1500-1800. Wandlungen einer Institution. Wilhelmshaven 1991, S. 40. Dieses Thema wird ebenfalls ausführlich beschrieben bei Schmuck, Peter: Der Wandel der Musikkultur als Phänomen des gesellschaftlichen Wandels am Beispiel der Innsbrucker Fürstenhöfe zwischen 1560 und 1650 - eine konstruktivische Analyse. Dissertation. Leopold Franzens Universität Innsbruck. Innsbruck 1999.

6 Ebd., S. 41. 
16. Jahrhunderts wanderte im Ergebnis der Säkularisierung der Kirchenmusiker ein Teil von ihnen aus der Sphäre zuvor üblicher Präbenden in die Position eines bezahlten Bediensteten am Hofe. ${ }^{7}$

Neben dem differenzierten Milieu des königlichen bzw. kaiserlichen Hofes mag diese relativ klare Bezeichnung weniger evident erscheinen, doch hier hilft vor allem ein gründliches Lesen der Quellen und ein Wissen um den Kontext: Als Instrumentalisten respektive musica wurden zuweilen auch Trompeter (und Kesselpauker), Posaunisten und ähnliche Musiker bezeichnet. Es ist folglich unstrittig, dass die spezialisierte Gruppe von Trompetern beispielsweise am Rosenberger Hof die Spitze der Repräsentation darstellte, doch lässt sich gerade von ihnen nicht von einem Typ die Norm übersteigender (außerhalb des Systems stehender) Bediensteter sprechen bzw. müssen diese auf eine Stufe mit Schauspielern gestellt werden; die musica besitzt ihre tiefen Wurzeln.

Aus Sicht der adäquaten Repräsentation erscheinen noch im Verlaufe des 17. Jahrhunderts mindestens zwei Trompeter an den fürstlichen und einigen gräflichen Höfen unverzichtbar (an letzteren häufig mit Blick auf die Funktion des entsprechenden Adeligen). Angesichts der Kostspieligkeit, über die sich im Übrigen gleich in der Einleitung der Instruktion Karl Eusebius von Liechtenstein äußert, stellt jedoch das musikalische Ensemble keinen gewöhnlichen, anspruchsvollen Bestandteil des adeligen Hofes dar. In dieser Hinsicht überschreiten die Musiker in der Tat den wirklichen Standard, abhängig in hohem Maße von den ökonomischen Möglichkeiten des Brotgebers, dessen Repräsentationsbedürfnissen und schließlich auch den persönlichen Vorlieben und Präferenzen; erst in diesem dritten Punkt finden wir eine Berührungsfläche mit dem Unterhalt einer adeligen Schauspielertruppe. Doch gerade der Vergleich zwischen Musiker- und Schauspielerensembles bzw. die Gegenüberstellung ihrer Präsenz an adeligen Höfen in der Frühen Neuzeit belegt das eindeutige Übergewicht der Musiker auch die angedeutete Notwendigkeit und Multifunktionalität derartiger Personen (insbesondere im Falle der Funktionen, der als musicus bzw. trometter bezeichneten Personen). ${ }^{8}$

Trotz der nicht unerheblich schwankenden Terminologie in der neuzeitlichen Historiographie lässt sich die übergroße Mehrzahl bestehender Musikensembles an den Höfen des böhmischen und mährischen Adels an der Wende vom 16. zum 17. Jahrhundert mit dem Terminus musica gleichsetzen. Für diese exaktere terminologische Erfassung plädiert beispielsweise Martin Horyna, der eingehend das Musikleben am Hofe der Rosenberger und in Krumau (Český Krumlov) selbst analysiert hat. ${ }^{9}$ Horyna verweist

7 Ebd., S. 53-60. Die Situation an den deutschen Höfen in der zweiten Hälfte des 16. Jahrhunderts dokumentieren eingehend vor allem Ruhnke, Martin: Beiträge zu einer Geschichte der deutschen Hofmusikkollegien im 16. Jahrhundert. Berlin 1963 und Schmid, Ernst Fritz: Musik an den schwäbischen Zollerhöfen der Renaissance. Beiträge zur Kulturgeschichte des deutschen Südwestens. Kassel - Basel - London - New York 1962.

8 Den Verlust der genauen Bedeutung der Termini trommeter/musicus konstatiert bereits für das ausgehende 16. Jahrhundert M. Grassl. Vgl. Grassl, Markus: Instrumentalisten und Instrumentalmusik am kaiserlichen Hof von 1527 bis 1612. In: Die Wiener Hofmusikkapelle III. Gibt es einen Stil der Hofmusikkapelle? (Hg. Hartmut Krones, Theophil Antonicek, Elisabeth Theresia Fritz-Hilscher). Wien - Köln - Weimar 2011, S. 109-148, hier S. 119.

9 Horyna, Martin: Vilém z Rožmberka a hudba (Wilhlem von Rosenberg und die Musik), Opera historica 3, 1993 [České Budějovice, Editio Universitatis Bohemiae Meridionalis], S. 257-264. Zur ältesten Geschichte 
dabei unter anderem auf grundlegende Parallelen zwischen der adeligen Musik und Ensembles städtischer Musiker (in der Regel eine Gruppe des Stadtturmes, Türmer bzw. Turmbläser). Im Jahre 1564 wirkten in der Rosenberger Musik lediglich sechs Musiker, deren Zahl stieg jedoch zum Ende des 16. Jahrhunderts von zehn auf zwölf Personen. Die Rosenberger ließen talentierte Knaben auf ihren Gütern bei kaiserlichen Musikern in Musik unterrichten, insbesondere in der Trompetenkunst. Musik erklang regelmäBig zum herrschaftlichen Mittags- und Abendmahl, Fanfaren ertönten vor allem bei Besuchen hochgestellter Personen (ebenso wie Turmbläser in den Städten). Drei Mal wöchentlich mussten die Musiker neue Kompositionen einstudieren, da „ihre Gnaden es zu bemängeln pflegte, wenn Stücke einfach häufig wiederholt wurden. "10

Vom eigenen Musikrepertoire am Hofe der Rosenberger zeugen die überlieferten musikalischen Inventare (1599, 1601, 1609, 1610), die ältesten Quellen dieses Typs aus dem adeligen Milieu der Länder der Böhmischen Krone, zu deren seltenen Parallelen insbesondere die Verzeichnisse liechtensteinischer Musikalien aus Prossnitz in Mähren (Prostějov) aus den Jahren 1607 und 1608 gehören. ${ }^{11}$ Die detaillierte Analyse dieser Dokumente liefert wertvolle Informationen hinsichtlich der Akquirierung des Repertoires und seines Transfers im adeligen Milieu, was u. a. das seltene Verzeichnis der Hinterlassenschaft des Grafen Julius von Salm auf Tobitschau (Tovačov; $\uparrow 1697$ ) belegt. ${ }^{12}$

Der erwähnte Peter Vok von Rosenberg beschäftigte neben Musikanten auch einen Hoforganisten und Diskantisten (jugendlichen Sänger). ${ }^{13}$ Eine schrittweise Reduzierung des ursprünglichen Rosenberger Ensembles lässt sich auch in der zweiten Dekade des 17. Jahrhunderts unter den Schwanbergern (Švamberkové) beobachten, als die Zahl der Musiker (noch für 1617 handelte es sich um sechs Trompeter und einen Organisten) sank und am Hofe Peters von Schwanberg nach 1618 lediglich noch zwei Trompeter belegt sind. ${ }^{14}$ Hoforganisten begegnen wir auch an weiteren führenden Höfen, unter anderem bei Karl von Liechtenstein, und zwar im wesentlichen in einem längeren Zeitraum,

des Rosenberger Musikensembles (Hofmusiker sind bereits im 15. Jahrhundert bezeugt) vgl. Ders.: Hudba a hudebni život v Českém Krumlově do poloviny 16. století (Musik und Musikleben in Krumau bis zur Mitte des 16. Jahrhunderts), Miscellanea musicologica XXXI, 1984, S. 286-287.

10 Mareš, František: Rožmberská kapela (Die Rosenberger Kapelle), Časopis českého musea 68, 1894, S. 209 236, hier S. 215. Die Ausgaben der Rosenberger analysierte eingehend Jan Schreib, wobei er jedoch die Anschaffung eines Zymbals (Metallinstrument des Glockentyps) mit der Akvirierung des Zymbals im Sinne eines Saiteninstruments verwechselte. Vgl. Schreib, Jan: K hudebnímu mecenátu posledních Rožmberki̊ (Zum Musikmäzenatentum der letzten Rosenberger), in: Opera historica 4, České Budějovice 1995, S. 77-89, hier S. 80 .

11 Maňas, Vladimír: Prostějouský inventár hudebnin z roku 1608 (Das Prossnitzer Inventar von Musikalien aus dem Jahre 1608). Opus musicum : hudební revue, Brno: Opus musicum, 2014, Jahrgang 46, Nr. 6, S. 6-29.

12 Racek, Jan: Inventář hudebnin tovačovského zámku z konce 17. století (Das Musikalieninventar des Schlosses Tobitschau vom Ende des 17. Jahrhunderts). Musikologie I, 1938, S. 45-68.

13 Mareš, F.: Rožmberská kapela, S. 220-221.

14 Kubeš, Jiř́: Švamberský dvưr v Třeboni v letech 1611/1612 až 1620 (Der Schwanberger Hof in Wittingau in den Jahren 1611/1612 bis 1620). Opera historica 7, 1999, S. 441-468, hier S. 465, Anm. 76. Wie zahlreiche andere Autoren benutzt auch Kubeš die allgemeine Bezeichnung kapela bzw. musika für dieses primär aus Trompetern bestehende Ensemble. 
als hier die eigentliche Musica wirkte. Organist und mitunter auch Sänger repräsentieren, ungeachtet der vermuteten, laufenden Vereinigung mit Instrumentalisten (an der Tafel etc. vertreten) jenen zweiten Hauptbestandteil der traditionellen höfischen Struktur, also die Kapelle. Zum terminologischen Wirrwarr trägt bei, dass auch die leitenden Instrumentalisten, die Musica, zuweilen in den Quellen als Kapellmeister bezeichnet werden $^{15}$, was die schrittweise Dekonstruktion der terminologischen Definition und im Verlaufe des 17. Jahrhunderts die fortschreitende Etablierung des Begriffes Kapelle als allgemeine Bezeichnung des Musikensembles vorwegnimmt.

In der ersten Hälfte des 17. Jahrhunderts jedoch sollten sich die Termini musica und capella (Kapelle) noch unterscheiden, wovon auch die unterschiedlichen Rollen der Musiker am Hofe Albrechts von Wallenstein Zeugnis ablegen. Die Verzeichnisse seines Hofes gestatten es leider nicht, die Entwicklung kontinuierlich zu verfolgen, zumal sie lediglich aus den Jahren 1625, 1630 sowie den nachfolgenden Jahren überliefert sind. Im Verzeichnis vom August 1625 figurieren lediglich zwei Trompeter mit einem Gehalt von 30 Gulden pro Musiker. In den Eintragungen vom Juli (?) und August 1630 gehörten zum herzoglichen Hof neben einem Tanzmeister und sechs Trompetern Musiker der Kapelle: neben einem Organisten und einem Orgeltreter (...) erscheinen hier sieben, im Augustverzeichnis nur mehr sechs Sänger. ${ }^{16}$ Die latinisierten Namen verraten leider mit Ausnahme des italienischen Tenors nicht deren Herkunft. Die ganze Gruppe ist unter der Bezeichnung Musicanten zusammengefasst, die Anwesenheit von zwei Dienern der Kapelle in der gleichen Kategorie unterstreicht klar die funktionale Einordnung der gesamten Gruppe. Im gleichen Dokument figurieren in der Einleitung eine Gruppe Stall Parthey die erwähnten Trompeter (Trommeter), so dass allgemein festgestellt werden darf, dass gerade das Juliverzeichnis den quantitativen Höhepunkt des herzoglichen Hofes bildet. ${ }^{17}$ In einem ähnlichen Verzeichnis vom Dezember 1630 fehlen die Musiker

15 In den liechtensteinischen Rechnungen aus dem Jahre 1604 wird der gerade neu angestellte Leiter der gräflichen Musica als Capellmeister bezeichnet. HAL, H 76, fol. 65r; vgl. des Weiteren Haupt, Herbert: Fürst Karl I. von Liechtenstein, Hofstaat und Sammeltätigkeit. Obersthofmeister Kaiser Rudolfs II. und Vizekönig von Böhmen. Edition der Quellen aus dem Liechtensteinischen Hausarchiv. Bd. 2. Quellenband. Wien - Graz 1983, S. 139.

16 Ulrich Cering Organista (22 Gulden 30 Kreuzern), Georg Hoffmann Bassista (15 Gulden), Fridericus Heilius Bassista (12, fehlt im nachfolgenden Verzeichnis von August), Henricus Franciscus Bassista (12, ebenso wie auch weitere erwachsene Sänger), Guilio Vera Tenorista, Gabriel Adalbertus Tenorista, Henningus Bonstadius Altista, Fridericus Daniel Discantista (6 Gulden). Národní archiv Praha, 67/33, Karton 47, fol. 57v, 65v. Die Namen der meisten Musiker mit fehlerhafter Datierung des Verzeichnisses auf Dezember (wenngleich es mit ziemlicher Sicherheit um die Zitierung aus dem Augustverzeichnis handelt) finden sich bei Schottky, Julius Max: Über Wallensteins Privatleben: Vorlesungen gehalten in dem Museum zu München. München 1832, S. 180.

17 Michal Konečný beziffert die Gesamtzahl der Angehörigen des wallensteinischen Hofes unter dem Hofmeister Paul Christoph von Liechtenstein-Castelkorn auf 273 Personen mit monatlichen Gesamtausgaben in Höhe von 3.953 Gulden. Vgl. Konečný, Michal - Vašek, Leoš: Kryštof Pavel z Liechtensteinu-Castelkornu a Morava v časech třicetileté války. Konečný, M. (Hg.): Christoph Paul von Liechtenstein-Castelkorn und Mahren in der Zeit des Dreisigjahrigen Krieges. Brno 2005, S. 15. Das analysierte Verzeichnis mit der höchsten Zahl an Musikern (insgesamt 8) und Trompetern (6), das vermutlich vom Juli 1630 stammt, umfasst jedoch 343 Personen und damit verbundenen monatlichen Gesamtausgaben von 5.011 Gulden 30 Kreuzern. Národní archiv Praha, Fond Valdštejniana Jičín, sign. 67/33, Karton 47, fol. 56r-62v. Zum Kontext des ökonomischen Hinterlandes der Karriere Wallensteins vgl. Kostlán, Antonín: Albrecht z Valdštejna jako 
der Kapelle bereits gänzlich, es tauchen lediglich zwei Bedienstete der Kapelle auf und die Zahl der Trompetern ist auf drei reduziert. ${ }^{18}$ Es darf also angenommen werden, dass auch im Falle Wallensteins das zahlenmäßige Anwachsen des Hofpersonals nicht einfach mit dem ökonomischen Aufstieg des Brotgebers - vor allem mit seiner Karriere und seinen Funktionen - zusammenhängt.

Gerade bei Wallensteins gibt es die Möglichkeit die praktische Betätigung von Musikern innerhalb ihrer höfischen Struktur (unter anderem in Abhängigkeit zur ausgeübten amtlichen Funktion des jeweiligen Adeligen) wie auch die theoretische Reflexion der Bedeutung der Musik und der Musiker am adeligen Hof anhand der Instruktion Karl Eusebius' von Liechtenstein zu verfolgen. In der nachfolgenden Interpretation folgen wir dessen ungeachtet der Chronologie, die es gestattet, die quantitative Situation an den Höfen der ersten Fürstengeneration der Liechtenstein, insbesondere im Falle Karls, die Veränderungen am Hofe seines Sohnes Karl Eusebius und schließlich auch die Situation im ausgehenden 17. Jahrhundert an den Höfen von Johann Adam Andreas und Maximilian Jakob Moritz von Liechtenstein zu vergleichen.

\section{Die Fürsten von Liechtenstein und Musik: erste Generation}

Was den Hof Karls von Liechtenstein (1569-1627) anbetrifft, hat die Gegenüberstellung unterschiedlicher Quellentypen eine Reihe wertvoller Informationen erbracht, die die Diskrepanzen zwischen Norm und Praxis bezeugen, aber auch den ausgeprägt wechselhaften Umfang der musikalischen Aktivitäten am liechtensteinischen Hof. Herbert Haupt verweist bereits für das Jahr 1599 auf den gräflichen Trompeter Michel, dem 24 Gulden gezahlt wurden, und auch in den nachfolgenden Jahrzehnten tauchen in den liechtensteinischen Schriftquellen verständlicherweise weitere Trompeter auf, wobei sich die Schlüsselfrage nach deren Einordnung innerhalb der höfischen Struktur stellt. Die meisten Hinweise finden sich in den liechtensteinischen Rechnungsbüchern, die mit Blick auf die erste Dekade des 17. Jahrhunderts - allerdings lediglich aus den Jahren 1604-1606 vorliegen. Eine selbständige Tätigkeit als Organist übte in den Jahren 1604 und 1605 ein gewisser Daniel Hoffmann aus, der ein Jahresgehalt von 35 Gulden bezog (weitere Organisten erscheinen erst in den nachfolgenden Jahrzehnten).

Zumindest in den Jahren 1604 bis 1606 beschäftigte Karl von Liechtenstein eine gröBere Zahl von Musikern, was möglicherweise mit seinem Amt als Landeshauptmann von Mähren (1604-1607) zusammenhängt. Im übrigen belegt die Anwesenheit der gräflichen Musica auf dem Landtag in Ungarisch Hradisch (Uherské Hradiště) im Sommer 1605 eine Ausgabe von vier Gulden für die Reise zu Pferde der führenden Musica und

investor do vlastního osudu. Ekonomické aspekty jedné raně novověké kariéry (Albrecht von Wallenstein als Investor in das eigene Schicksal. Wirtschaftliche Aspekte einer frühneuzeitlichen Karriere). In: Fučíková, Eliška - Čepička, Ladislav (Hg.): Albrecht z Valdštejna. Inter arma silent musae? Praha 2007, S. 38-61.

18 NA Praha, Fond Valdštejniana Jičín, sign. 67/33, Karton 47, Verzeichnis für Dezember 1630, fol. 141r-146v (abweichende Foliierung mit Bleistift). 
weitere zehn Personen von Hradisch nach Mährisch Aussee (Úsov). ${ }^{19}$ Es scheint daher wahrscheinlich, dass die Musiker auf diese Weise zu einem sicheren Ziel (Aussee repräsentierte zu dieser Zeit die nördlichste liechtensteinische Bastion in Mähren) vor der damaligen militärischen Grenze geschickt wurden.

Eine nähere Vorstellung über die Größe und Struktur des musikalischen Ensembles liefern bislang lediglich die erhalten gebliebenen Rechnungen. Am 27. November 1604 wurde der Schlosser für zwölf neue Schlüssel zur Kammer der Musiker (Music Jungen Cammer) auf Schloss Eisgrub (Lednice) entlohnt. Hier wohnten zusammen vermutlich zehn bis zwölf Musikgesellen, die schrittweise lernten, ein größeres Spektrum an Instrumenten zu beherrschen, wie dies damals bei professionellen Musikern gang und gäbe war; in den Rechnungen aus dem Jahre 1605 konnten dabei bislang neun Namen ermittelt werden. Im Mai desselben Jahres wurden beim Prossnitzer Schuster zwölf Paar Stiefel gerade für diese Gesellen gekauft. ${ }^{20}$ Das hohe jährliche Einkommen des liechtensteinischen Trompeters Hans Röttel (100 Gulden) könnte möglicherweise mit der Unterrichtung von Gesellen zusammenhängen. ${ }^{21}$ Große Erfahrungen mit der Leitung eines vokal-instrumentalen Ensembles besaß auch der liechtensteinische „Kapellmeister" Nicolaus Zangius (ca 1568-1617) selbst, nominell noch immer Kapellmeister an der Marienkirche der Hansestadt Danzig (1599-1607). Zu den liechtensteinischen Musikern gehörten auch Zangius' Diener Ludwig Raidl und zwei weitere Instrumentalisten, die für das Jahr 1606 Erwähnung finden: der Lautenspieler Peter Kapoun (Kappaun, Capaun), der neben einem Beitrag für Saiten auch eine goldene Kette im Wert von 50 Gulden erhielt, und der Zinkbläser (Zink, cornetto) Hieronymus. ${ }^{22}$ Im Unterschied zu anderen Instrumentalisten hat es jedoch den Anschein, dass beide keinen ständigen Lohn empfingen.

Wie aus den Rechnungen ersichtlich, war Zangius verantwortlich für den Kauf von Instrumenten und Noten, weshalb er im Herbst 1604 nach Wien reiste. Wenngleich er selbst komponierte, veröffentlichte er jedoch gerade in den Jahren 1604 und 1610 vermutlich keine umfangreiche Sammlung, sondern lediglich gelegentlich Hochzeitskompositionen $(1606,1608)$. Die nahezu identischen Inventare der liechtensteinischen Musikalien, stets zum 1. Januar 1607 und 1608 auf Schloss Prossnitz (Prostějov) verzeich-

19 Haupt, H.: Fürst Karl, S. 148.

20 HAL, Kart., H 76, fol. 49r.

21 Ebd., H 76. Vgl. den hohen Betrag für die Unterrichtung des Trompeters in der zweiten Hälfte des 17. Jahrhunderts im mährischen Milieu, der auf 100 Taler festgesetzt war. Vgl. Sehnal, Jiř́í: Trubači a hra na prírozenou trompetu na Moravě v 17. a 18. století (Trompeter und das Spiel auf einer natürlichen Trompete in Mähren im 17. und 18. Jahrhundert). In: Časopis Moravského muzea, vědy společenské, 73, 1988, S. 175-207; 74, 1989, S. 225-268, hier S. 176.

22 HAL, Kart. H 76. Kapouns Zugehörigkeit zum kaiserlichen Hof (vermutlich auf der Grundlage der Formulierung „ihrer Mt. Musico“ im Kontext des Geschenks jener Goldkette im Jahre 1606) erwähnt Haupt, H.: Fürst Karl, S. 60. Unter den Musikern am Hofe Kaiser Rudolfs II. konnte der genannte Kapoun bislang freilich nicht identifiziert werden. Vgl. Žáčková Rossi, Michaela: The Musicians at the Court of Rudolf II. The Musical Entourage of Rudolf II (1576-1612) Reconstructed from the Imperial Accouting Ledgers. Praha 2017 und Grassl, M.: Instrumentalisten. Die besondere Stellung des Zinkbläsers bei den Habsburgern untersucht Grassl in der Regierungszeit Ferdinands I. bis hin zu Rudolf II. (1574: leere Rubrik Zinkchenplaser), vgl. ebd., S. 120-121. 
net, enthalten neben gewöhnlichen Kategorien von Musikalien und Musikinstrumenten (zum Teil gerade durch Zangius erworben) auch ein eigenständiges Verzeichnis von Motetten (also geistlichen Kompositionen zu lateinischen Texten) des Nicolaus Zangius für sechs bis sechzehn Stimmen, die so zu einem Bestand der einzigartigen liechtensteinischen Musikalien wurden; leider sind sie jedoch nicht erhalten geblieben. ${ }^{23}$

Ähnlich wie bei Kompositionen für eine große Zahl an Stimmen, bei denen die Frage auftaucht, ob für deren Aufführung die liechtensteinische Musica wirklich eine ausreichende Zahl von Sängern zur Verfügung stand (diese Kompositionen entstanden vermutlich vornehmlich in der Zeit von Zangius' Wirken in der Danziger Stadtpfarrkirche, wo der Kapellmeister über eine ausreichende Zahl an Kräften verfügte), zeigen die Prossnitzer Inventare auch eine faszinierende Vielzahl an Musikinstrumenten, die bei weitem die gewöhnlichen Bedürfnisse eines adeligen Ensembles überstieg ${ }^{24}$, verzeichnet zusammen mit zwei Pauken und entsprechendem Zubehör (Quastenschnüre, Taftanhänge unter den Trompeten mit dem liechtensteinischen Wappen etc.) in einer gesonderten Aufstellung. Dem Ensemble standen darüber hinaus ein Regal mit zwei Virginalen, eine Unzahl von Blechinstrumenten (ohne Trompeten 71!) zur Verfügung; darunter sieben Posaunen, ein Dulzian und insgesamt 23 unterschiedliche Typen von Zinkbläsern. Für das Spielen im Freien nutzte man insbesondere Blechinstrumente mit doppeltem Rohrblatt und mit durchschlagendem Ton - Schalmeien, Krummhörner, Pommer und Rackette. Die eindeutige Ausrichtung der liechtensteinischen Musica auf Blechinstrumente wird auch beim Vergleich mit der Kollektion an Geigen und Violen deutlich, doch auch so ist deren Zahl hoch (drei Diskant- und eine fünfsaitige Bassgeige; sechs Violen da Gamba)..$^{25}$

23 Wenngleich das Verzeichnis nicht alle bekannten Kompositionen von Nicolaus Zangius enthält (in einigen Fällen führt es demgegenüber ansonsten unbekannte Kompositionen an), handelt es sich um eines der ältesten bekannten zeitgenössischen Verzeichnisse des Werkes eines konkreten Komponisten. Vgl. Maňas, Vladimír: Dobový soupis motet Nicolause Zangia z roku 1608 a jeho souvislosti (Das zeitgenössische Verzeichnis der Motetten des Nicolaus Zangius aus dem Jahre 1608 und sein Kontext). Opus musicum, Brno: Opus musicum, 2016, Jg. 48, Nr. 1, S. 6-21. Nahezu alle im Verzeichnis erwähnten Motetten für sechs Stimmen veröffentlichte Zangius im Jahre 1612 in Wien (und ein Jahr später in Leipzig) in der Sammlung Cantiones sacrae, gewidmet Hynek d. J. von Würben auf Freudenthal. Eine repräsentative Auswahl bietet die CD Nicolaus Zangius: Cantiones Sacrae. Ensemble Versus \& Capella Ornamentata. Brno 2017.

24 Im Vergleich zu den Inventaren der Rosenberger fällt die Zahl der Musikalien und Musikinstrumente unbestritten geringer aus, doch entstand die liechtensteinische Sammlung innerhalb einer im Grunde kürzeren Zeit und neben dem einmaligen Kauf in Wien, realisiert mit Hilfe des gräflichen Kapellmeisters Zangius und ortsansässiger Kaufleute, bezeugen einige Drucke, Handschriften und Instrumente die engen Beziehungen der Liechtenstein zum Kreis der kaiserlichen Musiker, vor allem zu Philippe de Monte und Carolo Luython. Vgl. Freeman, Michaela - David Freeman: Rare Instruments in the Bohemian Collections." In Musikalische Aufführungspraxis in nationalen Dialogen des 16. Jahrhunderts, Teil 2: Musikinstrumentenbau-Zentren im 16. Jahrhundert, 26. Musinstrumentenbau-Symposium Michaelstein, 6. bis 8 . Mai 2005, 237-60. Offenkundig wird, dass das Instrumentarium selbst auch gewisse repräsentative Züge besitzen konnte. Explizit ist eine solche Situation Mitte des 16. Jahrhunderts in Olmütz belegt, wo der städtische Rat den örtlichen Turmmusikern eine spezielle Kollektion von mit Gürtlerarbeiten geschmückten Instrumenten für außergewöhnliche Gelegenheiten im Rahmen der städtischen Repräsentation (Erneuerung des städtischen Rates, Eröffnung der Landtage) lieh. Vgl. Schlusche, Herbert: Stadtpfeifer und Instrumentenbauer in Olmütz im 16. Jahrhundert. Dissertation an der Karls-Universität Prag 1942. S. 8-9. 
Das zeitliche Ende dieser ersten liechtensteinischen Musica lässt sich nicht eindeutig ausmachen, vor allem aufgrund des Fehlens der Rechnungen aus den Jahren 1607 bis 1609. ${ }^{26}$ Im Jahre 1607 hielt sich Zangius nachweislich für kurze Zeit an seiner ursprünglichen Wirkungsstätte in Danzig auf. In den folgenden drei Jahren weilte er vermutlich auch in den böhmischen Ländern (u. a. in Prag und in Rossitz/Rosice) und er pflegte dabei mit Karl von Liechtenstein zumindest höflichen Kontakt, zugleich verschaffte sich Zangius Zugang zum weiteren Umfeld der Familie Žerotín. ${ }^{27}$ Im März 1610 vermittelte Zangius das Verleihen zweier liechtensteinischer Musiker an Johann Diviš von Žerotín („mir zwey Jungen, so guete Musici, weillen Sie die andere nit behanden haben, vergönnet“) und er sollte sich diesen auf Schloss Groß Seelowitz (Židlochovice), wo Žerotín den Fürsten Johann Georg von Jägerndorf beherbergte, selbst anschließen..$^{28}$ Die angedeutete Notlage mit Blick auf Musiker erscheint trotz der angenommenen Reduzierung doch überraschend. Im Januar und Februar 1610 war den liechtensteinischen Rechnungen zufolge der bereits erwähnte Musiker Ludwig Raidl zuletzt ausbezahlt worden („so bey fürstl. G. Für einen musicanten gedient"). Einmalig wird in diesem Jahr im Juli noch Peter Kapoun erwähnt, diesmal freilich mit einer Entlohnung in Höhe von 15 Gulden für die „Zusammenstellung des Ensembles“ („so er der musica zustellen soll“), mit größter Wahrscheinlichkeit bereitete also Kapoun selbst die musikalische Zusammensetzung für eine nicht näher beschriebene Festlichkeit bzw. eine andere Gelegenheit vor. ${ }^{29}$

Die nähere Beschreibung der liechtensteinischen Musica erwies sich als unerlässlich für die Gegenüberstellung der bislang bekannten Angaben aus den Rechnungsbüchern mit den ältesten Verzeichnissen des Hofstaates Karls von Liechtenstein, die Herbert Haupt erwähnt und die Thomas Winkelbauer eingehender analysiert hat. Aus der ursprünglich zahlenmäßig starken Gruppe von Musikern verblieben am liechtensteinischen Hof nach 1610 offenkundig nur Vítek Kř́istek ${ }^{30}$, Jiří Slavíkovský (als Kammerdiener wurden diese noch im Jahre 1617 entlohnt) sowie der erwähnte Mikuláš Rašek (Nicolaus Raschek), die in den liechtensteinischen Rechnungen bereits im Jahre 1605 unter der erwähnten Mu-

26 Eine detaillierte Übersicht zu den überlieferten Rechnungsbüchern Karls von Liechtenstein bietet Haupt, H.: Fürst Karl, II, S. 125.

27 Maňas, Vladimír: Musik am Hofe Karls I. von Liechtenstein zu Beginn des 17. Jahrhunderts im mitteleuropäischen Kontext. Eine Vermutung der Zusammenhänge. In: Die Liechtenstein und die Kunst. Vaduz: Verlag des Historischen Vereins für das Fürstentum Liechtenstein, 2014. S. 149-160, hier S. 157-158.

28 Zemský archiv v Opavě, pobočka Olomouc, Rodinný archiv Žerotínové Bludov (Landesarchiv Troppau, Zweigestelle Olmütz, Familienarchiv Žerotín Blauda), Buch Nr.19, Inv. Nr. 392, fol. 54.

29 Haupt, H.: Fürst Karl, II, S. 172, Nr. 164 (1610). Bis zum Jahre 1611 reicht der zuletzt erwähnte Kontakt von Zangius zu den Liechtenstein, der mit der Veröffentlichung (und einem möglicherweise Widmungsexemplar) einer weiteren Sammlung dreistimmiger deutscher Lieder des Komponisten in Wien im Jahre 1611 zusammenhängt: Am 29. Mai dieses Jahres wurde Zangius auf Weisung Maximilians von Liechtenstein (allerdings aus der Schatulle seines Bruders Karl) ein Lohn von 50 Gulden und 20 Kreuzern ausgezahlt (,auss befelh herrn Maximiliani herrn von Liechtenstein per welcher ihr g. ime verehrt haben“). Haupt, H.: Fürst Karl, II, S. 174.

30 Mit ziemlicher Sicherheit wurden gerade ihm 1611 zehn Gulden für eine Trompete sowie Saiten für Geigen ausgezahlt. Vgl. Haupt, H.: Fürst Karl, II, S. 174. 
sikgesellen (Music-Jungen) auftauchen. ${ }^{31}$ Lediglich dank der Kenntnis ihrer Namen lassen sich diese Musiker in den erwähnten Verzeichnissen aus den Jahren 1611/1612 und 1616 identifizieren. Der älteren Nachricht zufolge erhielt Raschek einen Jahreslohn in Höhe von 40 Gulden, drei weiteren Trompetern (neben Křístek und Slavíkovský wird hier auch ein gewisser Valentin Groß erwähnt) standen jeweils 20 Gulden sowie zusätzlich die gleiche Summe für Kleidung zu. Im Verzeichnis erscheinen sie hinter dem Kammerdiener (Camerling) Samuel Perger mit einem Jahreslohn von 100 Gulden, hinter den Trompetern folgt der Koch (Kuchlmeister) Andre Gostrelli mit einem Sold von 150 Gulden. ${ }^{32}$ Im jüngeren und zugleich umfangreicheren Verzeichnis figurieren die oben erwähnten drei Trompeter (ohne Valentin Groß) unter den Angehörigen „Cammerpartey“. ${ }^{33}$ Wesentlich erscheint, dass keines der Verzeichnisse des Hofstaates eine besondere Kategorie für Musiker aufweist, hingegen finden wir diese eingereiht in die gewöhnliche Struktur des Hofes, jedoch ohne die Identifikation ihrer Trompeterfunktion. ${ }^{34}$

Mit Blick auf die Musiker verkleinerte sich den Rechnungen zufolge nach 1618 der Hof Karls von Liechtenstein weiter. Die belegten Ausgaben für Musik betreffen somit in den zwanziger Jahren des 17. Jahrhunderts nahezu ausschließlich Organisten, in geringerem Umfang auch Trompeter (Feldtrompeter Hans Rehle für das Jahr 1623) und im Jahre 1625 wurde nach langer Zeit erneut der Lautenspieler Peter Kapoun entlohnt. ${ }^{35}$

Was die Brüder Karls von Liechtenstein anbetrifft, so erweisen sich die Nachrichten für Maximilian (1578-1643) als sehr sporadisch, wobei Informationen über Musiker bislang nicht aufgefunden werden konnten; im Falle Gundakers (1580-1658) machte Thomas Winkelbauer auf mehrere bemerkenswerte Situationen aufmerksam. Im Oktober 1631 beauftragte Gundaker seinen Kanzler Dr. Michael Schießel, wenn möglich einen Leiter der Musica („directorem musicae“) zu finden. ${ }^{36}$ Analog zur vormaligen Situation am Hofe Karls von Liechtenstein sollte dieser musikalische Leiter mit der Gründung eines ganzen Ensembles beauftragt werden. Eine unmittelbare Inspiration mochte für den Fürsten die feierliche Grundsteinlegung für die neue Pfarrkirche in Feldsberg (Valtice) im Herbst desselben Jahres gewesen sein. Bei dieser Gelegenheit trat nämlich ein Musikensemble (in den Rechnungen als musica bezeichnet) Kardinal Franz’ von Dietrichstein

31 In den Rechnungen tauchen sporadisch auch mit dem Kauf von Trompeten verbundenen Ausgaben auf: im Jahre 1610 zehn Gulden für ein Instrument für den Trompeter Vítek und im Jahre 161570 Gulden für eine silberne (!) Trompete. Haupt, H.: Fürst Karl, I, S. 61. Im Rechnungsmaterial findet des Weiteren die Bezeichnung Trompeter Erwähnung.

32 HAL, Kart. H 47, unfoliert.

33 HAL, Kart. H 2, unfoliert. Vgl. Winkelbauer, T.: Fürst und Fürstendiener, S. 356-358.

34 Die Institution des Orchesters als Musikensemble bildet sich erst im Verlaufe des 17. Jahrhunderts heraus, in den frühneuzeitlichen Quellen, insbesondere in Rechnungen, erscheint dieser Terminus wahrscheinlich erst seit dem 18. Jahrhundert. Das Ensemble von Instrumentalisten, das in der Oper musiziert, wird als Orchester erstmals 1702 erwähnt. Erst Johann Mattheson bezieht diesen Begriff auch auf Musikensemble außerhalb des Theaters. Vgl. seine Abhandlung Das Neu-Eröffnete Orchestre, Hamburg 1713 sowie weitere Traktate. Fukač, Jiří: Orchestr. In: Slovník české hudební kultury (hg. von Jiří Fukač, Jiří Vysloužil). Praha 1997, S. 664.

35 Für die Jahre 1622-1623 ist zudem der Organist Georg Sigl bezeugt, für 1625 sodann Tobias Justinidas. Vgl. Haupt, H.: Fürst Karl, I, S. 61, zu Kapoun Ders., Fürst Karl, II, S. 273.

36 Winkelbauer, T.: Fürst und Fürstendiener, S. 363. Das Konzept des Schreibens ist auf den 4. 10. 1631 datiert. 
auf, damals vermutlich das einzig funktionierende Ensemble dieser Art in Mähren. ${ }^{37}$ Weitere Indizien für die musikalische Begleitung am Hofe Gundakers von Liechtenstein stammen erst aus dem Jahre 1641; Ende März schickt der Wiener Hausmeister ein repariertes Regal (eine kleine, tragbare Orgel das nur aus aufschlagenden Zungenpfeifen besteht, und somit hat ein durchlagendes Ton.) ${ }^{38}$ und ein mit neuen Saiten versehene Violine zurück nach Mährisch Kromau (Moravský Krumlov). ${ }^{39}$

\section{Die Fürsten von Liechtenstein und Musik: zweite Generation}

Im Falle von Karl Eusebius von Liechtenstein (1611-1684) können dank der überlieferten Quellen mehrere miteinander verbundene Themen und Situationen verfolgt werden. Bei der erwähnten Feierlichkeit der Grundsteinlegung der Pfarrkirche in Feldsberg im Jahre 1631 erklangen möglicherweise Kompositionen der dietrichsteinischen Musiker Aloisi bzw. Abbate, beide Angehörige des Minoriten-Ordens. ${ }^{40}$ Carlo Abbate wird

37 Haupt, Herbert: Von der Leidenschaft zum Schönen. Fürst Karl Eusebius von Liechtenstein (1611-1684). Quellenband. Wien - Köln - Weimar 1998, S. 15: (8. 9. 1631): „... haben ihre fürst. Genaden fürst Carl Eusebio herrn cardinalds und fürstens von Dietrichstein musica, welche zu Veldtsperg, alss in der neüen kirchen der grundtstein gelegt worden, musiciret, verehren lassen $45 \mathrm{fl}$." Der Hinweis auf die Entlohnung des dietrichsteinischen Ensembles für einen stattgefundenen Auftritt widerspricht durch seine Datierung dem vermeintlichen Datum der Feierlichkeit, siehe Fleischer, Viktor: Fürst Karl Eusebius von Liechtenstein als Bauer und Kunstsammler (1611-1684). Wien - Leipzig 1910, S. 17-19, ableitete. Fleischers Angaben hat auch H. Haupt mit der Datierung der Feier auf den 23. 10. 1631 übernommen. Vgl. Haupt, H.: Von der Leidenschaft zum Schönen, S. 15. Unter den jüngeren Arbeiten vgl. Fidler, Petr: Die Maria Himmelfahrtskirche in Feldsberg. In Die Liechtenstein und die Kunst. Vaduz 2014, S. 275-294, hier S. 279 (26. 10.!). Auf den Widerspruch zwischen der Entlohnung für die musikalische Begleitung (für den 8.9. entlohnte Musiker, 20 feierliche Medaillen wurden am 2. 9. geprägt) und dem für gewöhnlich angegebenen Datum der Feierlichkeit hat bislang niemand aufmerksam gemacht.

38 Winkelbauer, T.: Fürst und Fürstendiener, S. 363. Genau dieses Regal ist möglicherweise das einzige, im Inventar der Schlosskapelle in Mährisch Kromau (1696-1706) erwähnte Musikinstrument. Ein Regal erscheint darüber hinaus in weiteren adeligen Instrumentarien an der Wende vom 17. zum 18. Jahrhundert (Tobitschau/Tovačov, Pirnitz/Brtnice, Krumau/Český Krumlov). Erst das Inventar aus dem Jahre 1709 verzeichnet neben einem Regal auch weitere Instrumente: drei Geigen, zwei Violas, ein Violon, drei Posaunen, zwei alte Cembalos, zwei Paar Pauken und zwei Waldhörner: „Violin drey, Pražen (!) zwey, Violon ein, Posaunen drey, alte flüegl zwey, Paukken zwey paar, Jäegerhorn zwey“. HAL, Kart. H 2574, fol. 267v; ältere Inventare ebd. fol. 329r u. a.

39 Im Falle des Violons handelt es sich möglicherweise um jenes identische Instrument, das nach dem Tode Hartmanns von Liechtenstein im Brünner Palais der Sekundogenitur (am Fröhlichertor) im Jahre 1686 Erwähnung findet. Vgl. MZA, C 2, Sign. L 12, Kart. 97, fol. 26r. Über dieses Palais eingehender Winkelbauer, T.: Fürst und Fürstendiener, S. 415. Den zitierten Informationen aus Gödls Topographie zum Trotz hat es den Anschein, dass sich das Haus auch in der ersten Hälfte des 18. Jahrhunderts im Besitz der Liechtenstein befand. Nach dem Tode des Fürsten Johann Adam wurden im Haus am Fröhlichertor u. a. ein unbenutzbares Klavichord und eine Harfe erwähnt. Vgl. MZA, C 2, Sign. L 53/1, Kart. 105, Fol. 382v.

40 Im Stadtarchiv Feldsberg (Valtice), untergebracht im dortigen Schloss, ist eine bemerkenswerte Handschrift überliefert, die den Titel Orgelbuch trägt. Es handelt sich um die Verpflichtung der Gemeinde zur Aufstellung einer Orgel mit zwölf Registern aus Holz und Zinn mit vier Gebläsen. Jiří Sehnal interpretiert dieses Dokument als Zeugnis des Aufbaus einer Orgel noch in der alten Pfarrkirche. Vgl. , viz Sehnal, Jiří: $\mathrm{Z}$ minulosti hudby ve Valticích (Aus der Musikvergangenheit in Feldsberg). In: Město Valtice (hg. von Emil Kordiovský). Valtice 2001, S. 339. Dem ursprünglichen Text zufolge handelt es sich um einen Plan zum 
im Jahre 1629 als Kaplan und Musiker des Fürsten von Dietrichstein tituliert. ${ }^{41}$ Giovanni Battista Aloisi, ein herausragender Musikkomponist, verknüpfte sein beachtenswertes Schicksal mit Mähren und der Familie Dietrichstein mit größter Wahrscheinlichkeit im Jahre 1631. ${ }^{42}$ Im Zeitraum 1628-1644 hatte er in Venedig insgesamt sechs Sammlungen lateinischer geistlicher Lieder (einschließlich einer Re-Edition) herausgegeben. Die Sammlung Corona stellarum duodecim widmete er mit dem Hinweis auf das sog. Loreto in Nikolsburg Anna Maria Fürstin von Dietrichstein, geb. von Liechtenstein (1597-1638), der Schwester von Karl Eusebius. Der Dedikation zufolge, datiert auf den 2. Februar 1637 in Nikolsburg, hatte Aloisi hier bereits fünf Jahre als Sekretär und Kapellmeister der Dietrichstein gewirkt. ${ }^{43}$ Die Kompositionen von Abbate und Aloisi waren den Inventaren zufolge in den Pfarrkirchen der liechtensteinischen Herrschaften (Mährisch Trübau/Moravská Třebová, Littau/Litovel, Goldenstein/Kolštejn, heute Branná) häufig vertreten. ${ }^{44}$

Bau einer neuen Orgel (deren Parameter sind hier lediglich in groben Zügen dargelegt, demgegenüber fehlt der entsprechende Name des Organisten bzw. der Disposition) im Wert von 800 Gulden. Angesichts der hohen Summe wurde eine öffentliche Sammlung ausgeschrieben und die erwähnte Handschrift stellt hier primär eine Übersicht der Spenden dar (an erster Stelle eine Spende des damaligen geistlichen Verwesers Nicolaus Hartmann in Höhe von 100 Gulden sowie weitere Spender). Daher erscheinen hier Personal und Höflinge der Fürstenresidenz, zugleich jedoch auch alle Einwohner (Hauswirte) von Feldsberg. Die genannte - scheinbar lediglich die musikalische Tätigkeit der Pfarrkirche betreffende - Quelle besitz also einen weitaus größeren Aussagewert. SOkA Břeclav mit Sitz in Nikolsburg (Mikulov), Archiv města Valtic, Inv. Nr. 78.

41 Vgl. Brázdová, Lucie: Hudba a kardinál Dietrichstein 1599-1636 (Die Musik und Kardinal Dietrichstein 1599-1636). Olomouc 2012, S. 144.

42 Im Nekrolog des Brünner Minoritenkonvents, in dem Aloisi am 20. 3. 1665 verstarb, wird er u. a. als Capellae Magister tituliert. Eingehender hierzu Tomaštík, Eduard: Giovanni Battista Alouisi - život a dilo (Giovanni Battista Aloisi - Leben und Werk). Dissertation am Institut für Musikwissenschaft der Philosophischen Fakultät der Masaryk-Universität Brünn, Brno 2012, S. 26. Vgl. des Weiteren auch Sehnal, Jiří: Italšti hudebnici na jižni Moravě v proni polovině 17. století (Italienische Musiker in Südmähren in der ersten Hälfte des 17. Jahrhunderts). Jižní Morava 2005, Jg. 41, Bd. 44, S. 83-96, hier S. 83-84. Der Minorit Aloisi war zudem Mitglied des Brünner Konvents zu St. Johannes, zu dem die Liechtensteiner eine starke Familientradition (Linie Boskowitz) unterhielten. Der Umfang des Mäzenatentums dieser beider Familien gegenüber den Brünner Minoriten ist bislang nicht eingehender untersucht worden. Im Falle von Karl Eusebius verdient insbesondere die Errichtung eines hölzernen, mit Silber verzierten Altars für den hl. Antonius von Padua, im Jahre 1660 in Auftrag gegeben, Aufmerksamkeit. Im Jahre 1665 bezahlte der Fürst für diesen einem Wiener Handwerker noch ausstehende fast 1150 Gulden, und noch im 1669 erhielt dann der Brünner Maler Johann Strobel für den Auftrag zur Errichtung des Altars, einschließlich der Vergoldung, 200 Gulden. Haupt, H.: Von der Leidenschaft zum Schönen, S. 76-77. An diesem Altar wirkte die gleichnamige, bereits 1647 gegründete - vom bischöflichen Konsistorium in Olmütz jedoch erst 1663 bestätigte- Antonius-Bruderschaft. Vgl. Malý, Tomáš - Maňas, Vladimír - Orlita, Zdeněk: Vnitřni krajina zmizelého města. Náboženská bratrstva barokního Brna (Die innere Landschaft der verschwundenen Stadt. Religiöse Bruderschaften im barocken Brünn). Brno 2010, S. 175.

43 Tomaštík, E.: Giovanni Battista Alouisi, S. 110-114.

44 Vgl. die Inventare aus Mährisch Trübau (1644) und Littau (1669 und später). Zu den liechtensteinischen Besitzungen gehörte auch Goldenstein (heute Branná). Im Verzeichnis der Musikalien der dortigen, zwischen 1677 und 1684 erbauten Pfarrkirche gehört auch ein nicht näher bestimmter Druck von Carlo Abbate. Zusammenfassend vgl. Sehnal, Jiř́: Hudebni inventár kostela v Moravské Třebové z konce třicetileté války (Das Musikinventar der Kirche in Mährisch Trübau vom Ende des Dreißigjährigen Krieges). Hudebni věda LII, 2015, Nr. 1, S. 5-28. 
Im Zusammenhang mit der Huldigung der Stände der Herzogtümer Troppau und Jägerndorf im Jahre 1632 in Troppau entstand offenkundig die älteste Reflexion des Hofstaates von Karl Eusebius von Liechtenstein. ${ }^{45}$ Die in tschechischer Sprache verfasste und noch im gleichen Jahr in Olmütz herausgegebene Relation des fürstlichen Regenten Johann Wenzel Sedlnický von Choltice ergänzt eine deutschsprachige Handschrift, vermutlich eine zeitgenössische Übersetzung der erwähnten Publikation. ${ }^{46}$ An der Spitze des reichen fürstlichen Gefolges fuhren zwei Trompeter. Bei der Einfahrt in die Stadt muss eine akustisch erstaunliche und außerordentliche Situation geherrscht haben: Vom Rathausturm und den Stadttoren spielten städtische Turmbläser, denen die Feldtrompeten der einzelnen Stände antworten: „In dießem ein Tritt haben sich die Stadt Thurner auf den Rath hauß, alß auch auff den andern Thor Thurmern stadtlich hören laßen, denen der herrn stände feldt trompetter auff dem Ring bey der Cavalleria daß obstatt garlustig gehalten, so seindt auch auf ein mahl undt alßo zu gleich in allen Kirchen die gloken bey einer gutten virtellstundt geleittet worden." ${ }^{47}$ Was die Rituale in der Pfarrkirche Mariä Himmelfahrt anbetrifft, geht besagte Relation auch auf die Qualität der Musikkompositionen ein: „so baldt haben sich die Musicanten mit der Orgel, Trompetten, zinken [,] kesselpaucken, posaunen, und allerhandt anderen Instrumenten hören laßen, mit völligem Chor daß Laudate Dominum omnes gentes! Dem allmächtigen Gott zu Ehren fröhlich auff gesungen." Die gesungene Messe folgte erst am darauffolgenden tag in der Frühe (12. 8.), danach gelobten die Stände dem Fürsten Gehorsam. Die Beschreibung des musikalischen Bestandteils erscheint in dieser Situation erkenntnisärmer. Im Falle der Pfingstsequenz Veni Sancte Spiritus (traditionell beispielsweise auch bei der Erneuerung des städtischen Rates gesungen) knüpften die Musiker an den Vorgesang des Erzdekans (,welches die Musicanten neben der orgel undt anderen schönen Instrumenten gesungen") an, bei der Messe selbst erklang dann der Hall der Trompeten und Pauken („nachdem ist daß hohe Ambt de Sancto Spiritu angehob, undt solcher gottes dienst zur Ehre gottes mit schöner Music [,] Trompetten, und Kesselpaucken aufs schönste cellebriret, undt begangen worden..“). Eine ähnliche Erwähnung betrifft auch den abschließenden Lobgesang Te Deum laudamus.

Die minimale Zahl von zwei Trompetern gehörte folglich zum Hofe von Karl Eusebius bereits bei dessen Erreichen der Volljährigkeit. Die erhaltenen Rechnungen, von Herbert Haupt exzerpiert, ermöglich in einem zeitlichen Längsschnitt eher zufällige Situation zu erfassen, bei denen Musiker entlohnt wurden, seien es nun die eigenen oder aber externen. ${ }^{48}$ Wenn wir nunmehr für einen Augenblick das repräsentative Ausmaß

45 Eingehender zu diesem Ereignis vgl. Müller, Karel: Huldigung an Karl Eusebius, Fürst von Liechtenstein im Jahr 1632 in Troppau, In: Vařeka, Marek - Zářický, Aleš (Hg.): Das Fürstenhaus Liechtenstein in der Geschichte der Länder der Böhmischen Krone. Ostrava - Vaduz 2013, S. 179-186.

46 Die Beschreibung des Fürstenhofes von Karl Eusebius übersetzt und interpretiert Haupt, H. (Hg. Kräftner, Johann): Fürst Karl Eusebius von Liechtenstein, S. 52-59. Zum Druck der Sumarni relace (Olomouc 1632) vgl. HAL Wien, Kart. H 500, die deutsche Übersetzung ebd., MS. 913.

47 HAL Wien, MS. 913 (unfoliert). Haupt vergisst in seiner Darstellung die Anwesenheit der Feldtrompeter des Adels. Vgl. Haupt, H. (hg. Kräftner, Johann): Fürst Karl Eusebius von Liechtenstein, S. 56. Zur Problematik der Feldtrompeter vgl. insbesondere Sehnal, J.: Trubač (Die Trompeter).

48 Die Situation am Wiener Hof unter den Kaisern Ferdinand II. und Ferdinand III. fasste zusammen Seifert, Herbert: Eine Krise der Hofkapelle unter den Kaisern Ferdinand II. und Ferdinand III.?. In: Fritz-Hilscher, 
des Hofes und seiner Bestandteile außer Acht lassen, dann muss eingeräumt werden, dass nicht einmal der fürstliche Hof mit seinen Bedürfnissen ein ständiges Musikensemble unterhalten musste. Es macht also Sinn, über eine gewisse Dichotomie zwischen der langjährigen Beschäftigung von Trompetern (potenziert darüber hinaus durch deren Multifunktionalität, vor allem die Kombination der vermuteten Bildung sowie der militärischen Erfahrungen betreffend ${ }^{49}$ ) und dem gelegentlichen Bedarf eines größeren Ensembles nachzudenken. ${ }^{50}$

Im Übrigen genügte, wie aus den Rechnungen ersichtlich, für private Aufführungen eine kleinere Anzahl von Musikern, zugleich muss jedoch betont werden, dass die Perspektive des Rechnungsmaterials eher außergewöhnliche Ereignisse und die Produktion ausländischer Musiker in den Mittelpunkt rückt (ähnlich wie im Falle von Theatervorstellungen etc.). ${ }^{51}$ So tauchen im Jahre 1641 (mit Datierung auf den 31. 12. in Wien) in den Rechnungen eine relativ hohe Entlohnung von sechs Gulden für zwei Musiker, vier Gulden für den Stallmeister und Türsteher Swatkowsky (vielleicht auch im Sinne der Musikproduktion) und sechs Dukaten für einen leider namentlich nicht genannten italienischen Musiker auf. ${ }^{52}$ Im Jahre 1675 war für einen nicht näher verifizierten Auftritt auch der Sänger Nicolao Grancini entlohnt worden (9 Gulden, bezeichnet als „musicant"). ${ }^{53}$ Vermutlich handelte es sich um einen Kastraten, da ein Sänger dieses Namens in den Jahren 1668 bis 1670 am herzoglichen Hof in Württemberg auftrat und am Ende

Elisabet Theresia - Krones, Hartmut -Antonicek, Theophil (Hg.): Die Wiener Hofmusikkapelle II. Krisenzeiten der Hofmusikkapellen. Wien - Köln - Weimar 2006, S. 99-12.

49 Im Falle von Karl Eusebius bietet sich zum Beispiel nachfolgender Rechnungsbeleg aus dem Jahre 1644 an: „[...] dem trometter Simon Heinz zu unterhalt [...] eines pferts, so herrn von Zinzentorff nach Wien geschickt worden, und verpflegung aines englischen hunts, so er trommeter von grafen von Hardegg bringen soll, geben 5 fl. $r$. 30 kr.", Haupt, H.: Von der Leidenschaft zum Schönen, S. 41. Der Trompeter begleitete auch den Verwalter der liechtensteinischen Gestüte auf dessen Weg nach Mantua im Jahre 1679, ebd., S. 251. Im Falle von Johann Adam Andreas von Liechtenstein sicherte dessen Trompeter beispielsweise den Transport von sechs Pferden von Plumenau (Plumlov) nach Dobrau (Nové Zámky) im Jahre 1686. Vgl. Haupt, H.: Ein Liebhaber der gemähl und virtuosen... Fürst Johann Adama I. Andreas von Liechtenstein (1657-1712). Wien Köln - Weimar 2012, S. 471.

50 Innerhalb der Struktur des kaiserlichen Hofes ist für das Jahr 1637 erstmals die Unterscheidung von musikalischen und nichtmusikalischen Trompetern bezeugt: tubicines (non) musicales, vgl. Grassl, M.: Instrumentalisten, S. 118-119. Die sog. musikalischen Trompeter, zwischen acht und elf Personen, einschließlich eines Kesselpaukers, gehörten zur Gruppe der kaiserlichen Instrumentalisten und dank des Lohnes im Rahmen des höfischen Musikensembles stand ihnen ein höherer Lohn als den übrigen Trompetern und Kesselpaukern zu. Vgl. Seifert, H.: Eine Kris,e S. 103.

51 Diese Perspektive wird noch gesteigert im Falle der Auswahleditionen von Herbert Haupt, die prinzipiell Einzelfälle stärker als den normalen Betrieb des Hofes sichtbar machen: wie bereits angedeutet, bleiben die Musiker darüber hinaus häufig innerhalb des Dienstpersonals im Verborgenen. Vgl. auch Haupt, H. (hg. von Kräftner, Johann): Fürst Karl Eusebius von Liechtenstein, S. 79.

52 Haupt, H.: Von der Leidenschaft zum Schönen, S. 35, Nr. 317: „... zween musicanten 6 fl. $r$, herrn stallmeister und aufwarter Swatkowski, so sie in ihr maytt. thiergarten zu verehren hergeliehen, 4 fl. r., dann ein Wallischen, wegen ihrer f. g. gewießener spiel 6 ducaten ...".

53 Ebd., S. 95. Im gleichen Jahr erhielt ein gewisser Baptista Braxanela (Bazanela), der drei Frauen aus Italien mitgebracht hatte („drey weiber auss Wällischlandt“), wohin er sie am Ende auch zurückführte, vom Fürsten zwei hohe Saläre. Auch in diesem Falle könnte es sich um Sängerinnen gehandelt haben. Ebd., S. 95 . 
durch den Kastraten Marchesini ersetzt wurde. ${ }^{54}$ Für das Musizieren an der Tafel („,vor taffel musicirt") wurden im Januar 1668 zwei, beim zweiten Mal im gleichen Monat drei Studenten aus Prag entlohnt.

In ähnlichen Situationen jedoch dominierten in Schloss Feldsberg offenkundig lokale Musiker, die vornehmlich zur Karnevalszeit herangezogen wurden. So werden für das Jahr 1639 jüdische Musiker erwähnt, im Jahre 1671 spielten wiederum drei jüdische Musiker beim Karneval („,bey gehaltener mascara“). ${ }^{55}$ Mehrfach wurden „örtliche“ Musiker entlohnt (unerwähnt bleibt, ob es sich um städtische oder jüdische Künstler handelte), im Jahre 1668 konkret für die Musik zur Karnevalszeit. ${ }^{56}$ Ähnlich wie im Falle der nicht zu vernachlässigenden Entlohnungen für das Spiel auf der Fiedel (1665: „leyerin zu Wien“ 1 Gulden 30 Kreuzer) und eine Fiedlerin mit Trommler (1675, 3 Gulden 30 Kreuzer) ${ }^{57}$ dürfte es sinnvoll sein, über ein zielgerichtetes Ausscheren aus den üblichen ästhetischen Rahmenbedingungen nachzudenken. Insbesondere bei Karnevalsproduktionen konnten sich die höchsten Kreise am deftigen Spiel der Musiker aus unteren Schichten erfreuen, was unter anderem andere beliebte Karnevalsvergnügungen am Hofe des Olmützer Bischofs Karl von Liechtenstein-Castelcorn (1664-1695) bezeugen. ${ }^{58}$

Bei Gottesdiensten griff der Fürst zumeist auf lokale Kirchenmusiker zurück: Feldsberger werden so im Jahre 1661 erwähnt, als sie für ein Stück in der Schlosskapelle bei einem Hochzeitszeremoniell und bei Frühmessen (rorate) Honorare erhielten. ${ }^{59}$ Bei Frühmessen, die traditionell bereits vor der Dämmerung begannen, sollten die Musiker den ganzen Advent über spielen. Die vereinzelte Verlegung dieser traditionellen Adventssitte in die Fürstenkapelle mochte zwar mit der fortschreitenden Rekonstruktion der Feldsberger Pfarrkirche zusammenhängen, ähnliche Entlohnungen für örtliche Musiker tauchen in den Rechnungen jedoch auch unter dem Fürsten Johann Adam Andreas auf. ${ }^{60}$ Aufmerksamkeit verdient die Verlegung einiger Gottesdienste in die Pfarrkirche St. Jakob in Brünn, beispielsweise anlässlich der Taufe von Johann Adam Andreas von Liechtenstein im Jahre 1657 (den Einzug des Prinzgefolges nach Austerlitz sicherte dann der Trompeter Georg Jabkensky ${ }^{61}$ ) oder der Hochzeit der Tochter Eleonore Marie Rosalie mit Johann Siegfried Fürst von Eggenberg im Jahre 1666. Feierlichkeiten fanden darüber hinaus im Kaunitz-Palais in Brünn statt, für musikalische Darbietungen wurden

54 Owens, Samantha: The Rise and Decline of Opera at the Württemberg Court, 1698-1733. In Italian Opera in Central Europe. Volume 1: Institutions and Ceremonies (hg. von Melania Bucciarelli, Norbert Dubowy, Reinhard Strohm), Berlin 2006, S. 99-114, hier S. 101.

55 Haupt, H.: Von der Leidenschaft zum Schönen, S. 88.

56 Ebd., S. 62, 64, 84. Die Feldsberger jüdische Musica entging bislang der Aufmerksamkeit der Forschung. Eingehender hat sich mit diesen Musikern, die für gewöhnlich in dreiköpfiger Besetzung auftraten, Jiří Sehnal am Beispiel des dietrichsteinischen Nikolsburg gewidmet. Vgl. Sehnal, J.: Židovské tanečni kapely na Moravě (Jüdische Tanzkapellen in Mähren), Hudební věda 34, Nr. 3, (1997) S. 292-302.

57 Haupt, H.: Von der Leidenschaft zum Schönen, S. 77, 94.

58 Sehnal, J.: Pavel Vejvanowský and the Kroměrižz music collection, Olomouc 2008, S. 207-229, v. a. S. 222 f.

59 Haupt, H.: Von der Leidenschaft zum Schönen, S. 68.

60 Haupt, H.: Ein Liebhaber der gemähl und virtuosen, S. 25 (1686); namentlich erwähnt wird hier der Schulmeister Johann Franz Sinacius), S. 53 (1690), S. 140 (1698).

61 Pospichal, B.: Zu einer richtschnuer, S. 20. 
Trompeter („denen sammentlichen trommetern, stadt musicanten, taffel deckhern...“), Stadtmusiker (also die städtische Musica, Gruppe der städtischen Turmwache) und vielleicht auch Musiker aus den Reihen der Dienerschaft entlohnt. ${ }^{62}$

Die Instruktionen von Karl Eusebius setzen im Falle der liechtensteinischen Prinzessinnen eine umfassende musikalische Bildung voraus, die eine Annahme von mehreren Instrumenten, Gesang und kompositorischen Grundlagen umfassen. ${ }^{63}$ Vereinzelte Nachrichten aus den Rechnungen, die ein Musizieren in der Fürstenfamilie selbst belegen, betreffen allerdings lediglich die Beschaffung und Reparatur von Instrumenten (erst in der nachfolgenden Generation verzeichnen die Rechnungen auch individuellen Musikunterricht). Ähnlich wie im Falle der Produktion verschiedenartiger Jagdhörner wandte sich der Fürst vorrangig an Wiener Hersteller von Musikinstrumenten. ${ }^{64}$ Der Lautenmacher Marcel Hellmayer lieferte so in den Jahren 1648-1651 eine damals beliebte Gitarre für die Fürstin Johanna Beatrix und versorgte sie wiederholt mit neuen Saiten. ${ }^{65}$ Auf dem gleichen Instrument spielte auch die Prinzessin Eleonora, was der kauf von Saiten in Prag im Jahre 1664 bezeugt. ${ }^{66}$ Interesse verdient darüber hinaus auch die Erwähnung des Kaufs eines Paars kleiner Pauken für Johann Adam Andreas im Oktober 1663 für zwölf Gulden und 15 Kreuzer ${ }^{67}$ Egal, ob wir nun unter den Pauken ein spezifisches Spielzeug oder ein Musikinstrument verstehen, im Fall des - von einer musizierenden Familie umgebenen- sechsjährigen Prinzen erscheint dies wie ein vorteilhaftes Geschenk.

62 Haupt, H.: Von der Leidenschaft zum Schönen, S. 78. Gerade in der Funktion des Truchsesses (Taffeldecker) erscheinen an den Adelshöfen auch Musiker. Am Hofe des Grafen Johann Adam von Questenberg war der Truchsess (Michael Walter und später Karel Míca) traditionell für das Einstudieren von Komödien verantwortlich. Vgl. Perutková, Jana: Der glorreiche Nahmen Adami. Johann Adam Graf von Questenberg (1678-1752) als Förderer der italienischen Oper in Mähren. Wien 2015, S. 309-311. Musiker erscheinen an diesem Hofe unter anderem auch in der Funktion des Türstehers (Aufwarter), ebd. S. 329, was wiederum auf ältere Traditionen verweisen mag. Der „Tafeldecker“ des Grafen Rudolf von Gašín in Hultschin (Hlučín) Frantz Jacobi (cca 1673-1752) wurde nach dem Tod des Grafen ebd. Leiter des Chores in der Pfarrkirche und kaiserlicher Steuereinnehmer, vgl. Maňas, V.: Hudba v Hlučině v období raného novověku (Musik in Hultschin in der Frühen Neuzeit). Opus musicum 2002, Jg. 34, Nr. 5, S. 4-12. An der Wende vom 17. zum 18. Jahrhundert wirkte der Viola da Gamba-Spieler in der Funktion des Tafeldeckers im oberösterreichischen Kloster St. Florian, vgl. Lindner, Andreas: Musik im Stift St. Florian: Der soziale Status des Musikpersonals im 18. Jahrhundert. Studien zur Musikwissenschaft, 48. Bd. (2002), S. 321-371, hier S. 353. Derartige Einzelbeispiele finden sich sehr häufig, die Frage bleibt. Inwiefern sich hieraus Erkenntnisse generalisieren lassen. Zum breiteren Kontext von Musikensembles in der ersten Hälfte des 18. Jahrhunderts mit Hinweis auf den Unterschied zwischen Musikern-Dienstleuten und freien Hofkünstlern (hofmusici) vgl. Kapsa, Václav: Hofmusici a lokajové. K postaveni hudebnika na šlechtickém dvoře v Čechách proni poloviny 18. století. (Hofmusiker und Laien. Zur Stellung des Hofmusikers am Adelshof in Böhmen in der ersten Hälfte des 18. Jahrhunderts), Theatrum historiae 6, Nr. 9 (2011), S. 241-255.

63 Pospichal, B.: Zu einer richtschnuer, S. 89-90.

64 Jagdhörner (und offenkundig auch Trompeten) lieferte an den liechtensteinischen Hof insbesondere der Wiener Hersteller von Trompeten und Posaunen Johann Geyer, vgl. Haupt, H.: Von der Leidenschaft zum Schönen, S. 65. Ein Ensemble von neun bzw. acht unterschiedlichen Jagdhörnern erscheint in den Inventaren der liechtensteinischen Garderobe, verzeichnet in Feldsberg in den Jahren 1678 und 1684. Von deren repräsentativer und Signalfunktion zeugt die Tatsache, dass weitere Instrumente hier nicht auftauchen. Vgl. Haupt, H.: Von der Leidenschaft zum Schönen, S. 415-416, 481.

65 Ebd., S. 50, 53, 54.

66 Ebd., S. 73.

67 Haupt, H.: Von der Leidenschaft zum Schönen, S. 71. 
Was die Musica im Sinne eines fürstlichen Ensembles selbst anbetrifft, bietet sich für die lange Regierungszeit von Karl Eusebius von Liechtenstein selbstverständlich als Vorbild das durch den Komponisten Nicolaus Zangius aufgebaute und geleitete Ensemble an. Dessen nachweisbares Wirken fällt jedoch noch in die Zeit vor der Geburt Karl Eusebius'. Darüber hinaus bot in der zweiten Dekade des 17. Jahrhunderts Zangius eher zumindest den liechtensteinischen Trompetern eine zeitweilige Wirkungsmöglichkeit, im Falle des Nikolaus Raschek schließlich sogar am kurfürstlichen Hof in Berlin, wo Zangius an seinem Lebensende als Kapellmeister wirkte (1612-1617). In den nachfolgenden Jahrzehnten sind Nachrichten über liechtensteinische Trompeter eher sporadisch überliefert, von einigen fürstlichen Trompetern war bereits im Zusammenhang mit der Huldigungsreise nach Troppau die Rede. Für das Jahr 1655 gehörte zum fürstlichen Hof ein bereits zahlenmäßig stärkeres Ensemble von sechs Trompetern und einem Kesselpauker, das am Ende der siebziger Jahre noch um zwei weitere Trompeter anwachsen sollte. ${ }^{68}$ Erwähnung verdient, dass von einem neunköpfigen Ensemble bereits die Hofstaatsordnung Ferdinands I. von Habsburg aus dem Jahre 1527 ausging. ${ }^{69}$

Mit Ausnahme der kostspieligen Jagdhörner ${ }^{70}$ haben sich aus der Regierungszeit von Karl Eusebius offenkundig keinerlei Verzeichnisse erhalten, die den Inventaren aus Prossnitz aus den Jahren 1607 und 1608 ähneln würden. Über die Tatsache, dass diese ursprünglich reichhaltige Sammlung von Musikalien und Musikinstrumenten aller Wahrscheinlichkeit im Dreißigjährigen Krieg verloren ging, als auch das Schloss Prossnitz in Flamme aufging, zeugt indirekt auch das Inventar der liechtensteinischen Garderobe aus dem Jahre 1658. Zumindest mit Hilfe unerlässlicher ornamentaler Ergänzungen wird somit die Existenz eines Ensembles von Trompeten und Kesselpauken belegt („14 trompeten und heerpaukhen fahnen von schwarzen topeltaffet, darauf das l. wappen und flamme gemahlet"), das wesentlich bescheidener als jenes Prossnitzer Ensemble ausfiel. ${ }^{71}$

68 Winkelbauer, T.: Fürst und Fürstendiener, S. 365. Das Verzeichnis aus dem Jahre 1655 nennt die Namen der meisten Bediensteten. Der erste Trompeter Johann Franckenberger erhielt ein Jahresgehalt von 240 Gulden, die übrigen drei Musiker jeweils 180 Gulden (Georg Jabkensky, Adam Angermayer, Michael Fohl), Hieronymus Mayer sollte 150 Gulden erhalten, Johann Guttenberger 100 Gulden (möglicherweise hingen diese Gehälter avonden Erfahrungen im Dienst ab), an letzter Stelle erscheint der Kesselpauker Michael Mayer mit einem Gehalt von 80 Gulden, vgl. HAL Wien, Kart. H 47, unfoliert. Franckenberger erwähnt die bereits nachträglich durchgestrichene Anmerkung im Zusammenhang mit einer Trompete mit Goldschmuck im Inventar aus dem Jahre 1633; vgl. Haupt, H.: Von der Leidenschaft zum Schönen, S. 346.

69 Grassl, M.: Instrumentalisten, S. 119, noch nach dem Jahre 1545 sollte das Ensemble von Trompetern am Hofe Ferdinands I. acht bis neun Mitglieder umfassen; ebd.

70 Haupt, H.: Von der Leidenschaft zum Schönen, S. 415-416.

71 Haupt, H.: Von der Leidenschaft zum Schönen, S. 387. Es handelt sich zweifellos um Trauerbehänge, eingerichtet im Zusammenhang mit den Prager Exequien für den verstorbenen Karl von Liechtenstein; vgl. „item in 13 trometen fanen guldene und silberne wappen sambt flammen undt umb einen guldenen strich zu mallen, von einem stuckh 6 fl. r. “(1628), Haupt, H.: Von der Leidenschaft zum Schönen, S. 11-12. 


\section{"das flagollet ist auch so lieblich..." - Karl Eusebius über Musik}

Die wiederholt erwähnte Instruktion Karl Eusebius' an seinen Sohn enthält ungemein wertvolle Passagen über die Musik als Bestandteil der fürstlichen Repräsentation, aber auch über den Musikbetrieb selbst. Die grundlegende, den finanziellen Anspruch des Musikensembles (hier wird jener zweckmäßige Terminus Musica benutzt) und die Vorteilhaftigkeit der einzelnen Instrumente betreffende Passage wurde bereits veröffentlicht, erfuhr aber bislang nur geringe Aufmerksamkeit. ${ }^{72}$

Dies belegt unter anderem auch die These des Kenners der liechtensteinischen Geschichte, Gustav Wilhelms, der die Errichtung eines Theaters in Feldsberg am Ende des 18. Jahrhunderts als erst zweiten Höhepunkt der musikalischen Aktivitäten an den Höfen der Liechtenstein (als ersten derartigen Gipfel bewertet er das Wirken von Zangius) bewertet. ${ }^{73}$

Den Vorstellungen von Karl Eusebius zufolge sollten eine Musica maximal zwölf exzellente Musiker bilden: in dieser Zahl inbegriffen einen Organisten, einen (!) Sänger und ansonsten Geiger, zumal Lauten und Geige vor allem in größerer Zahl einen angenehmen Ton erzeugen, vorteilhaft sowohl für die Kapelle als auch zum Tanz bzw. bei Spaziergängen (!). ${ }^{74}$

Die übrigen Instrumente wie Pfeifen, Posaunen und andere sind bei weitem nicht derart angenehm: „so viell eher ein khierren, als lieblichkeit causiren." Einen angenehmen, süßen Ton schreibt der Fürst zudem noch einem Instrument mit der Bezeichnung ,flageolet" zu, das vor allem in Frankreich (und England) im 17. Jahrhundert den beliebten Typus einer einfachen Flöte darstellte, der in mitteleuropäischen Quellen nur sehr vereinzelt Erwähnung findet. ${ }^{75}$ Gerade diesem eher instruktiven Instrument mit einem begrenztem Tonumfang und feinem Klang, typisch für den privaten Gebrauch, widmet

72 Haupt, H. (hg. von Kräftner, Johann): Fürst Karl Eusebius von Liechtenstein, S. 268-269. Der entsprechende Teil über die Musik beginnt mit folgender Passage:

„... viell fürsten halten ein Musica zu welcher wir dir weder rathen, noch müßrathen wollen, eher aber müßrathen, dann es seynd nur leith so gar viell kosten wan sie guth seyn, und also grosse unkosten und um so viell mehrers bediente, deren bedienten gar zu grosse zahl, viell ungelegenheit verursachet wan du aber fellig au $\beta$ deinen schulden wehrest, und die zeiten gar guth, keine krieg und andere ungebegenheiten, so kuntest eine halten, von ein 12 persohnen auf meyste, welche aber gar excellent seyn müsten in denen stim[m]en, und instrumenten [.]" HAL Wien, Signatur Hs 462.

73 Wilhelm, Gustav: Die Fürsten von Liechtenstein und ihre Beziehungen zu Kunst und Wissenschaft. Jahrbuch der Liechtensteinischen Kunstgesellschaft 1976, Vaduz 1977, S. 31. Hannes Stekl verfolgt das Musikgeschehen in Feldsberg erst seit der Gründung der sog. Harmoniemusik im ausgehenden 18. Jahrhundert. Vgl. Stekl, Hannes: Harmoniemusik und „türkische Banda“ des Fürstenhauses Liechtenstein. In: Haydn Jahrbuch 10 1978, S. 164-175. Vgl. auch Sehnal, J.: Z minulosti hudby ve Valticich (Aus der Vergangenheit der Musik in Feldsberg), S. 337, vgl. auch S. 353, Anm. 1

74 Vgl. Pospichal, B.: Zu einer richtschnuer S. 87-88, als ideale Anzahl nennt der Autor unter Bezugnahme auf Karl Eusebius jedoch irrtümlich 18 Musiker.

75 Einen kurzen Abschnitt widmet diesem Instrument in seinem Wörterbuch der Prager Musiker und Theoretiker Tomáš Baltazar Janovka, der freilich praktisch nur das Ausmaß des Instruments behandelt (hier flagolet). Vgl. Janovka, Tomáš Baltazar: Clavis ad thesaurum magnae artis musicae (hg. von Jiř́ Motl). Praha 2006, S. 96-99. Was die mährischen Quellen anbetrifft findet sich bislang keine andere Erwähnung eines „flageolet“. Für wertvolle Hinweise dankt der Autor Jana Perutková, Jiří Sehnal und Tereza Žůrková. 
sich das Buch paradoxerweise am ausführlichsten. Spätere Reflexionen erwähnen das Flageolett häufig als Instrument zum Training von Vögeln im Gesang, im 18. Jahrhundert sollte gerade das Flageolett den Gesang von Vögeln evozieren. ${ }^{76}$ In den fürstlichen Rechnungen finden sich gerade die Ausgaben für Singvögel und aufwendige Käfige für diese sehr häufig. Karl Eusebius von Liechtenstein pflegte dem Flageolett gegenüber offenkundig eine persönliche Beziehung, zumal er detailliert dessen Verwendung in einzelnen Situationen erläutert: es erweist sich wenig geeignet für vielstimmige Kirchenmusik, doch lassen sich durch dieses geistliche Lieder begleiten (bislang konnte keine mögliche literarische Vorlage dieser Passage gefunden werden, und die zeitgenössische Literatur erwähnt dieses Flageolett für gewöhnlich nur sehr kurz): „das flagollet ist auch lieblich, so sonsten zwar in kein cappelln tauget, es konte aber dennoch gebrauchet werden zu litaneyen und Salve Regina, Weihnachts und oster lieder zu singen."77

Erst nach diesem Exkurs widmet sich der Fürst wiederum allgemeineren Dingen: Die Singstimmen sollten von Kastraten besetzt werden („und seynd zum singen die Walschen die besten" $)^{78}$, als Geiger empfiehlt er jedoch einen Franzosen oder Deutschen. Ähnlich wie im falle des Flageoletts (mitunter auch als flautino francese bezeichnet) ${ }^{79}$ und der allgemeinen Präsenz der Geigen zeigt sich hier eine Inklination zu französischen Vorbildern, einschließlich der alternativen Auffassung der Musica gerade als gemischtes Ensemble. Dieses taucht neben einem Blechensemble am französischen Königshof bereits unter Franz I. auf, am kaiserlichen Hofe in Wien entstand ein solches Ensemble im Rahmen der Hofmusik im Jahre $1623{ }^{80}$ Die Musica bildet für den Fürsten ein vor allem für gottesdienstliche Zwecke geeignetes Ensemble (ganz im Gegensatz zu seiner höfischen Herkunft), erst danach lässt sich über deren Verwendung an der Tafel bzw. beim Promenieren nachdenken: „da du aber ein Musicam halten thätest, so sollest du solche besonders zu der ehr gottes halten, sie zugebrauchen in der Cappellen, und der kirchen und nachmahl erst zur delectation bey der taffl und der Promenade, sonsten seynd die trompetten genug und angenehm."

Die Musica erscheint dem Fürsten also wahrlich als ein luxuriöses Ensemble, zulässig lediglich in einer Situation, in der der Aristokrat völlig frei von Schulden ist und die Zeit günstig erscheint, während von Trompetern ganz automatisch als notwendiger Bestandteil des Hofes gesprochen wird. In der Auffassung von Karl Eusebius handelt es sich bei der Musica um jenen veränderlichen Typ eines Musikensembles, der sich praktisch

76 Pascual, Beryl Kenyon de - Waterhouse, William: Flageolet. Grove Music Online. www.oxfordmusiconline. com/grovemusic/view/10.1093/gmo/9781561592630.001.0001/omo-9781561592630-e-0000042044 (aufgerufen am 21. 7. 2018).

77 HAL Wien, Signatur Hs 462. Die Erwähnung der Weihnachtsfeiertage als Zeitraum, in dem man das Flageolett nutzen konnte, entspricht der spezifischen Verwendung von Blockflöten-Flöten in der Kirchenmusik im Sinne eines Hirteninstruments im Repertoire von Pastorellen. Vgl. Sehnal, J.: Pavel Josef Vejvanovský, S. 112.

78 Von hier stammt auch eine der ersten Reflektionen der fürstlichen „Kapelle“, die „mit denen castraten und musicis mehrer überhäufft als besetzt.“ Walaschek von Walberg, Theobald F. A.: Genealogie des durchlauchtigsten Hauses von Liechtenstein und Nikolsburg (1775), Kap. 3, Anm. 3. Zitat bei Haupt, H. (hg. von. Kräftner, Johann): Fürst Karl Eusebius von Liechtenstein, S. 179, Anm. 112.

79 Janovka, T. B.: Clavis ad thesaurum, S. 97.

80 Seifert, H.: Eine Krise, S. 104. 
gemäß den aktuellen Tendenzen aus Sicht der Besetzung und des Repertoires formiert. Die proklamierte (inwieweit jedoch realisierte?) Pflicht zu liturgischer Musik verknüpft diese mit der Tradition früherer Ensembles der Kapelle, durch das Bedürfnis nach einem weltlichen Repertoire lässt sich diese Auffassung der Musica mit der veränderten Bedeutung des Terminus Kapelle gleichsetzen. ${ }^{81}$ In der Instruktion des Fürsten findet praktisch die Musik zum Tanze keinerlei Erwähnung, Bedarf und Nachfrage waren dabei unstrittig vorhanden. Insbesondere Karnevalskompositionen waren in der zweiten Hälfte des 17. Jahrhunderts sehr beliebt. Im Zusammenhang mit dem bereits erwähnten Engagement verschiedener externer Musiker (jüdische, städtische, Kirchenmusiker, reisende Virtuosen) darf festgestellt werden, dass gerade diese die vorhandenen Bedürfnisse saturierten.

Demgegenüber ist für die fürstliche Repräsentation das Ensemble von Trompetern mit einem Kesselpauker absolut unerlässlich. Im Übrigen können diese Musiker sowohl zur Tafel, als auch „auf der Reys“ im Sinne der zu akzentuierenden fürstlichen Stellung (siehe Intrade als ursprüngliche Terminus für musikalische Kompositionen mit normalerweise zeremoniellem Charakter) musizieren, notfalls auch für die Dressur von Pferden. Karl Eusebius zufolge benötigen zahlreiche Fürsten, Adelige und Generäle Trompeten und Trommeln eher zum Bankett, doch erscheint es notwendig, Mäßigung im Essen und Trinken anzustreben (und sich nicht als schicksalhaftes, in einem Fürstenhause unerträgliches Laster anzugewöhnen). Der Fürst hat somit Trompeten auf fürstliche Weise zu nutzen, „[...] die trompetten ist ein Martzialisches Instrument, aber auch zugleich fürstlich, und bey ihren höfen gebräuchlich, danenhero du solches auf die fürstl: arth gebrauchen sollest" und den regierenden Herren der Fürstenhäuser stehe es nicht an am Kriege teilzunehmen. $^{82}$

Während Karl Eusebius in seiner Erklärung der Musik in freier Form zur Belehrung über die fürstliche Würde schreitet, erscheint es notwendig, kurz den Begriff des Trompetenblasen und dessen Stellung in der adeligen Gesellschaft des 17. Jahrhunderts darzulegen. Die scharfen Grenzen zwischen den einzelnen Ensembles - Musica und Trompeter - bildet nicht die Musik selbst (deren Charakter und Repertoire), sondern gerade die Ausnahmestellung und zeitgenössische Auffassung des Trompeters.

Der Adept eines Feldtrompeters musste ein freier Mann sein (und ein Zeugnis für seine eheliche Herkunft vorlegen können). Die entscheidende Barriere (oder besser einen Zensus) stellte die hohe Taxe (100 Taler im ganzen Reich) für eine zweijährige Ausbildung dar. Die Aufwendungen hierfür übernahm in der Regel der Adelige, bei dem der Trompeter am Ende zumindest für eine gewisse Zeit Tätig auf der Grundlage einer Übereinkunft war. Als Garantie überreichte der Trompeter dem Arbeitgeber seinen Lehrbrief. Spätestens sieben Jahre nach der Lehre musste der Trompeter an einem Feldzug teilnehmen, erst diese Erfahrung berechtigte ihn, selbst Lehrjungen zu unterrichten.

81 Eine unerlässliche terminologische Beschreibung, die insbesondere für das 18. Jahrhundert zutrifft, liefert Kapsa, Václav: Hudebnici hraběte Morzina. Př́spěvek $k$ dějinám šlechtických kapel v Čechách v době baroka (Die Musiker des Grafen Morzin. Ein Beitrag zur Geschichte adeliger Kapellen in Böhmen in der Barockzeit). Praha 2010, v. a. S. 14-17. 
So entstand eine relativ schmale berufliche Gruppe, die ihren Dienste als freie ritterliche Kunst auffasste (im Unterschied zu den städtischen Musikern, die eher durch Handwerker geprägt wurden). ${ }^{83}$ Allerdings existierte auch innerhalb dieser Gruppe offenkundig eine innere Differenzierung, die die erwähnte Unterscheidung kaiserlicher Trompeter in Musiker und Nichtmusiker charakterisiert. Eine größere Zahl an Trompetern findet sich in Mähren primär an den Fürstenhöfen. Neben der Familie Liechtenstein geht es in erster Linie um den Hof des Fürsten Karl von Liechtenstein-Castelkorn, dessen Ensemble von Trompetern Jiří Sehnal detailliert beschrieben hat. An der Spitze dieser Musiker stand ein Jesuitenschüler, der Komponist Pavel Josef Vejvanovský, dessen Titel eines Hof- und Feldtrompeters auch ein Zeitgenosse in Frage stellte. Vejvanovský und zahlreiche weitere bischöfliche Trompeter erwarben als Absolventen jesuitischer Gymnasien eine breite Bildung und beherrschten auch Latein. ${ }^{84}$ Spätestens seit dem Jahre 1664 besaß auch die mährische ständische Repräsentation einen eigenen Trompeter. Den Beschlüssen des Landtags in diesem Jahre zufolge hat es den Anschein, dass dieser primär als offizieller Bote diente, also in einer ähnlichen Funktion agierte, wie sie die Trompeter und Kesselpauker auch im Heer innehatten. ${ }^{85}$ Erst im Jahre 1702 gründeten die mährischen Stände ein Ensemble von Landestrompetern und Kesselpaukern (vier Trompeter und ein Kesselpauker). Die Entstehung dieser Landeseinrichtung entspricht der schrittweisen Reduzierung der Zahl der Trompeter an Adelshöfen in der ersten Hälfte des 18. Jahrhunderts. ${ }^{86}$

Die erwähnte Multifunktionalität der höfischen Trompeter, die mit deren angenommener Bildung und den militärischen Erfahrungen zusammenhing, bleibt auch im Falle

83 Sehnal, J.: Trubač a hra (Der Trompeter und das Spiel), S. 185-178.

84 Eingehender hierzu Sehnal, J.: Pavel Vejvanovský, S. 126-152. Wenngleich sich in narrativen Quellen häufig Hinweise auf sechs bischöfliche Trompeter finden (beispielsweise bereits im Jahre 1664 beim Einzug des Bischofs in Olmütz, bei dieser Gelegenheit soll es hier auch zwölf weitere Trompeter des mährischen Adels gegeben haben). Den Rechnungen zufolge lässt sich während des Episkopats die Beschäftigung von vier Trompetern und einem Kesselpauker nachweisen. Vgl. Sehnal, J.: Trubači a hra (Trompeter und das Spiel), S. 181

85 Die älteste bislang bekannte Nachricht stammt aus dem Jahre 1644 aus dem Landtagsprotokoll: „da ihre Gnaden die Herren Stände ursprünglich einen Landestrompeter zu unterhalten pflegten, den sie in Diensten des Landtags hierhin und dorthin senden konnten, soll auch fortan ein solcher Trompeter unterhalten werden, als einen solchen haben sie dann Herrn Johann Christoph Kampa verpflichtet und ihm jährlich 300 Gulden (,), einen Trompeterrock sowie eine Quastenschnur zur Trompete bewilligt, der, wenn er in Diensten des Landes entsandt wird, 45 Kreuzer am Tag erhalten soll.“ MZA, fond Stavovské rukopisy (A 3), Inv. Nr. 22, fol. 383v. Diese Informationen verdankt der Verfasser Jiří David. Die oben angeführte Formulierung verweist dessen ungeachtet primär auf die Funktion eines repräsentativen Boten bzw. Gefolges. Dem entsprechen auch die Pflichten des Brünner Turmwächters entsprechendes des Vertrages von 1674, demzufolge einige musikalische Pflichten im Zusammenhang mit der Landesrepräsentation (Trompete und Pauke spielen vom Turm des Rathauses bei einer Sitzung des Landtags) gerade dem städtischen Trompeter oblagen. Sehnal, J.: Trubači a hra (Trompeter und das Spiel), S. 192.

86 Vgl. Sehnal, J.: Trubači a hra (Trompeter und das Spiel), S. 179, 192 sowie ältere Studien von Bohumír Štědroň, relevant vor allem für das 18. Jahrhundert. Štědroň, Bohumír: Zemští trubači a tympanisté v Brně. K vývoji neznámé hudebni společnosti v 17. a 18. století (Landestrompeter und Kesselpauker in Brünn. Zur Entwicklung einer unbekannten Musik-Gesellschaft im 17. und 18. Jahrhundert). Vlastivědný věstník moravský 7, 1952, Nr. 3, S. 122-134, Nr. 4, S. 167-192. Ders.: Zur Entwicklungsgeschichte einer unbekannten Musik-Gesellschaft im 17. und 18. Jahrhundert. Die Musikforschung 21, 1968, S. 438-458. 
der Instruktion unter der Oberfläche verborgen, wobei diese ohne Zweifel jedoch einen Anteil an jener unbedingten Anwesenheit des Trompeter-Ensembles am Fürstenhofe besitzt. Der beachtenswerte Detailexkurs des Fürsten zu Umfang und Bedeutung des kleinen Instruments mit Namen Flageolett deutet an, dass zahlreiche Zusammenhänge bislang noch im Verborgenen sind. Zu diesen Details, die in gewisser Weise mit der Musik zusammenhängen, gehört die auch Tatsache, dass trotz des bekannten Rückzugs Karl Eusebius' vom kaiserlichen Hof der Fürst im November 1668 beim kaiserlichen Kapellmeister Antonio Bertalli (1605-1669), eines unter anderem hervorragenden Geigers und Autors zahlreicher Kompositionen für Violine, die jährliche schwindelerregende Summe von 20.000 Gulden lieh. ${ }^{87}$

\section{Die Fürsten von Liechtenstein und Musik: dritte Generation}

Die oben erwähnte Instruktion verdient eine Konfrontation mit den bisherigen Erkenntnissen über die Musik (und Trompeter) innerhalb der dritten Generation der liechtensteinischen Fürsten. Im Falle von Johann Adam Andreas von Liechtenstein (1657-1712) belegt das Rechnungsmaterial, wenngleich der Fürst vornehmlich in Wien residierte, die Kontinuität von Adventmessen in der Feldsberger Schlosskapelle, bei denen örtliche Kirchenmusiker agierten, das Auftreten eines externen Ensembles (beim Karneval im Jahre 1697 spielten in Feldsberg zwölf Eggenberger Musiker ${ }^{88}$ ) und die Erziehung der Fürstenkinder (Cembalo- und Flötenunterricht). ${ }^{89}$

Ein einzigartiges Dokument verkörpert vor allem das Verzeichnis der Musikalien, das am 22. 12. 1691 und am 7. 6. 1692 aus der Hinterlassenschaft des verstorbenen liechtensteinischen Kapellmeisters Johann Friedrich Lamb $(\uparrow 1691)$ entnommen und dem (fürstlichen) Organisten anvertraut wurde. Es handelt sich hier bei weitem nicht allein um Lambs eigene Kompositionen, sondern eher um seine Sammlung von Musikalien, die Sonaten (19), vier Messen, eine Motette, Kompositionen zu Vespern, aber auch eine

87 Haupt, H.: Von der Leidenschaft zum Schönen, S. 187: „Schuldverschreibung des Fürsten Karl Eusebius von Liechtenstein für den kayser. Capellmeistern Johann Anton Bertalli für ein Jahr auf die Summe von 20.000 Gulden“ (Nr. 1546, 4. 11. 1668). Das Grundgehalt des Kapellmeisters Bertalli betrug 1.200 Gulden, mit weiteren Zahlungen und dem Lohn für seine Gemahlin, ebenfalls einer Musikerin, lag das Jahreseinkommen bei 2.000 Gulden, im Jahre 1651 zahlte ihm Kaiser zudem die einmalige Summe von 3.000 Gulden. Vgl. Knaus, Herwig: die Musiker im Archivbestand des kaiserlichen Obersthofmeisteramtes (1637-1705). Bd. II, Wien 1968, S. 129, 132.

88 Haupt, H.: Ein Liebhaber der gemähl und virtuosen, S. 128. Entlohnungen für Musiker anlässlich von Karnevalsauftritten finden auch später Erwähnung, für gewöhnlich handelte es sich um hohe Summen auch angesichts einer größeren Zahl von Musikern (24 Musiker für das Jahr 1707) als auch mit Blick auf den zeitlichen Umfang (mehrere Tage) der Feierlichkeiten. Vgl. ebd., S. 182.

89 Ebd., vgl. u. a. S. 131-132, 194. Mit der Übersiedlung des Fürsten nach Wien verlor Feldsberg zwar seinen Residenzcharakter, doch blieb dessen zentrale Funktion im Rahmen der Verwaltung der fürstlichen Besitzungen erhalten, ebenso der Verbleib einiger Dienstleute des Hofes. Der fürstliche Trompeter Johannes Rundt $(\dagger 1714)$ wurde am Ende sogar um 1700 Mitglied des städtischen Rates. Vgl. Valeš, Tomáš - Konečný, Michal: Umělci a umělečti řemeslníci ve valtických matrikách 17. a 18. stoleti (Künstler und Kunsthandwerker in Feldsberger Matrikeln des 17. und 18. Jahrhunderts). Opuscula historiae artium. 2011, Jg. 60 [55], Nr. 1, S. 50-73, hier S. 52. 
Sammlung italienischer Arien und weitere Noten enthält. ${ }^{90}$ Dieser bislang nicht näher bekannte Komponist widmete dem Grafen Julius von Salm in Tobitschau (Tovačov) ${ }^{91}$ eine eigene Messe (Missa Ferdinandi, die im erwähnten Verzeichnis nicht auftaucht) und wahrscheinlich sein Magnificat (im Verzeichnis sind zwei Kompositionen dieses Titels aufgeführt) um 1675 Pavel Josef Vejvanovský in Kremsier (Kroměříž) kopierte. ${ }^{92}$

Im Falle von Gundakers Enkel Maximilian Jakob Moritz Fürst von Liechtenstein (1641-1709) liefert das Musikalieninventar des Schlosses Tobitschau, das nach dem Tod des erwähnten Grafen Julius von Salm angelegt wurde, wertvolle Zeugnisse zu den Kontakten und dem Transfer des Repertoires. Eine der zahlreichen anonymen Ballette, also instrumentaler Stücke, die nicht allein zum Tanz dienten ${ }^{93}$, hatte Graf Salm als Geschenk „a Principe Stanicensi“ erworben. Maximilian Jakob Moritz von Liechtenstein hatte nämlich ursprünglich auf dem südmährischen Schloss Steinitz (Ždánice) residiert, dem Zentrum einer umfangreichen Herrschaft; allerdings konnte bislang lediglich die Anwesenheit fürstlicher Trompeter nachgewiesen werden. ${ }^{94}$ Im Jahre 1672 verlieh der Olmützer Bischof Karl von Liechtenstein-Castelkorn dem Fürsten Maximilian zwei seiner Trompeter zum Zwecke eines Feldzuges, bei dem es um die grundlegende Möglichkeit zur Gewinnung notwendiger militärischer Erfahrungen ging. ${ }^{95}$

Maximilian Jakob Moritz von Liechtenstein übersiedelte nach dem Tod seines Vaters Hartmann im Jahre 1686 nach Mährisch Kromau. ${ }^{96} \mathrm{Zu}$ Beginn des 18. Jahrhunderts ist eine Ausstattung der Schlosskapelle mit Instrumenten bezeugt, das höfische Rechnungsmaterial seit 1697 führt darüber hinaus auch Musiker an. Mit einer gewissen Vereinfachung und auf der Grundlage eines Vergleichs mehrerer Verzeichnisse aus dem Untersuchungszeitraum, einschließlich des Verzeichnisses der Empfänger von Deputat-Bier ${ }^{97}$,

90 Haupt, H.: Ein Liebhaber der gemähl und virtuosen, S. 532-533.

91 Racek, J.: Inventář hudebnin (Das Inventar der Musikalien), S. 53, 61. Die nicht näher bestimmte Komposition (zu Vespern?) erscheint in den Inventaren des Zisterzienserklosters in Ossegg (Osek) in den Jahren 1706 und 1720; vgl. Renton, Barbara Ann: The Musical Culture of Eighteenth-century Bohemia, with Special Emphasis on the Music Inventories of Osek and the Knights of the Cross, Dissertation New York 1990, S. 501, 509. Vgl. des Weiteren Sehnal, J.: Pavel Vejuanovský, S. 85.

92 Vgl. Sehnal, Jiří - Pešková, Jitřenka: Caroli de Liechtenstein-Castelcorno episcopi Olomucensis operum artis musicae collectio Cremsirii reservata, Pragae 1998. Vol. I, S. 333. Lambs Magnificat weist eine relativ einfache Besetzung für vier Solostimmen, zwei Geigen, Orgel und einen vierstimmigen Chor auf, ebd. Überliefert ist sie auch im Musikalieninventar der Moritzkirche in Kremsier aus dem Jahre 1695 („Magnificat Aut: Lamb), ebd., S. 57.

93 Brewer, Charles E.: The Instrumental Music of Schmeltzer, Biber, Muffat and their Contemporaries, Ashgate 2011, S. 181.

94 Racek, J.: Inventář hudebnin (Das Musikalieninventar), S. 66: No. 11: „Ballettae a 6... a Principe Stanicensi.“

95 Sehnal, J.: Trubači a hra (Die Trompeter und das Spiel), S. 187-188.

96 Kroupa, Jiří: Proměny moravskokrumlovského zámku v době renesance a baroka (Die Veränderungen des Schlosses in Mährisch Kromau in Renaissance und Barock). In: Fišer, Zdeněk (Hg.): Moravský Krumlov ve svých osudech. Brno 2009, S. 270. Zum Aufenthalt des Fürsten in Steinitz vgl. zusammenfassend Vrbas, Jakub: Ždánsko (Die Region Steinitz). Ždánice 1930, S. 291-292.

97 Es handelt sich um ein überraschend aussagekräftiges Material, wie am Beispiel des im Besitz der Questenberger befindlichen Jarmeritz (Jaroměřice) Perutková, Jana: Der glorreiche Name, v. a. S. 549-551, 555, aufzeigen konnte. 
lassen sich die hier erwähnten Musiker in zwei Gruppen einteilen. ${ }^{98}$ Auf der einen Seite handelt es sich um Personal der Pfarrkirche und der Schule (Lehrer, zwei Kirchenmusiker - Trompeter und zugleich Sänger, sog. Stadtorganist), den engeren Hof präsentieren sodann sechs Hoftrompeter und ein Kesselpauker ${ }^{99}$, der Hoforganist und seiner Fürsorge anvertraute drei jugendliche Sänger. Die Frage bleibt, ob zu den Musikern nicht auch weitere Bedienstete des Hofes gehörten (Kammerdiener, Tischbedienstete). Die vermutete enge Zusammenarbeit zwischen Schloss- und Kirchenmusikern wird durch besagtes Register bezeugt, darüber hinaus darf angenommen werden, dass sich im Milieu einer Kleinstadt die gleichen Musiker auch um die liturgische Musik in der Kirche des dortigen Paulinerklosters kümmerten. ${ }^{100}$

Unter Maximilian Jakob Moritz fanden in Mährisch Kromau auch Theateraufführungen statt. Im November 1703 wurde im Schloss das Stück Die Gedruckte Doch nicht unterdruckte Unschuld Agnetis aufgeführt, das Libretto gab noch im gleichen Jahr der Wiener Drucker Leopold Voigt heraus. ${ }^{101}$ Die Aufführung hing mit den häufigen Besuchen des Theatermachers Heinrich Rademin nicht allein in Kromau selbst, sondern auch in der königlichen Stadt Brünn zusammen. ${ }^{102}$ Diese bislang einzigartige Nachricht aus dem Beginn des 18. Jahrhunderts lässt sich auch als Vorbote der reichen (Musik-)Theateraktivitäten

98 MZA, Velkostatek Moravský Krumlov (F 177), Inv. Nr. 2702-3, Karton 802 (1697-8).

99 Die Trompeter (Veith Trübner, Franz Hellmann, Franz Marsch, Jacob Plessner, Wenzl Kraus, Jacob Kuczera) werden in einigen Fällen auch mit ihren Gesellen sowie mitunter auch den Söhnen genannt. Die meisten dieser Namen erwähnt auf der Grundlage eines genauen Exzerpts der Matrikeln in Mährisch Kromau Hlaváček, und zwar auch für frühere Zeiten. Vgl. Hlaváček, Petr: Hudba v Moravském Krumlově (Die Musik in Mährisch Kromau). In: Fišer, Zdeněk (Hg): Moravský Krumlov ve svých osudech. Brno 2009, S. 284-285. Unter den Matrikeleintragungen erwähnt Hlaváček auch die Titulatur eines fürstlichen Kapellmeisters: Im Jahre 1691 starb ein gewisser Ferdinand Lampel und nach 1700 (zuletzt 1707) wird mit dieser Bezeichnung auch der vormalige städtische Organist Jan Kroupa bezeugt, der zuletzt 1723 Erwähnung findet - wiederum als städtischer Organist. Nach dem Tod des Fürsten Maximilian Jakob Moritz erfolgte zumindest eine Reduzierung des Ensembles von Trompetern auf zwei. Petr Hlaváček erwähnt darüber hinaus auch den kaiserlichen Hof- und Feldpaukenspieler (Kay. Hoff- undt Feldt Pauker) Jakob Leopold Helmann, der im Jahre 1730 von seiner verwitweten Mutter das väterliche Haus erbte; ebd., S. 285. Eine mögliche prosopographische Untersuchung könnte unter Hinzuziehung der Deputat-Register und weiterer Quellen aus dem Familienfond und insbesondere des Großgutes weitere interessante Angaben auch mit Blick auf die Familienbeziehungen unter dem liechtensteinischen Personal zutage fördern. So war beispielsweise Anton Lux, der sich vor 1718 um inen Platz als Tenor der Kollegiatkirche St. Peter und Paul in Brünn beworben hatte (Sehnal, J.: Z minulosti hudby ve Valticich, S. 337) und ausschließlich mit seiner Stimme Fürst Maximilian in Mährisch Kromau dienen sollte, mit ziemlicher Sicherheit der Sohn des dortigen Bildhauers J. C. Lux, vgl. Valeš, T. - Konečný, M.: Umélci a umělečtt řemeslníci (Künstler und Kunsthandwerker), S. 61.

100 Paulín Joseph Mayer war darüber hinaus auch Verfasser der Grabpredigt im Jahre 1709 (Das in der Finsternuß Leichtende Liecht ... Das ist: Der ... abgelebte ... Maximilian / Jacob / Moritz ... Fürst von= und zu Liechtenstein ... mit einer Lob= und Leichen=Rede angezeiget. Wien: Andreas Heyinger). Vgl. Oratio Funebris. Die katholische Leichenpredigt der frühen Neuzeit. Zwölf Studien. Mit einem Katalog deutschsprachiger katholischer Leichenpredigten in Einzeldrucken 1576-1799 aus den Beständen der Stiftsbibliothek Klosterneuburg und der Universitätsbibliothek Eichstätt (edd. Birgit Boge - Ralf Georg Bogner). Amsterdam 1999, S. 157.

101 Bohatta, Hans: Das Theaterwesen am Hofe des Fürsten von und zu Liechtenstein. Jahrbuch der Gesellschaft für Wiener Theaterforschung 1950/51, Wien 1952, S. 38-86, hier S. 39.

102 Havlíčková, Margita: Brněnská účinkování Heinricha Rademina (Die Brünner Theaterauftritte Heinrich Rademins). Theatralia 2007, Jg. 56, Nr. Q10, S. 17-36, hier S. 18. 
an den Höfen der Fürsten von Liechtenstein und deren Patronaten in den beiden nachfolgenden Jahrhunderten begreifen. ${ }^{103}$

\section{Fazit}

Der doch außerordentlich umfangreiche Bestand der liechtensteinischen Besitzungen und des fürstlichen Archivs selbstzählt zu den Hauptgründen für die bislang relativ geringe Reflexion des Musikgeschehens an den Höfen der Fürsten von Liechtenstein. Eine gewisse Kontinuität lässt sich vor allem im Bemühen, einen eigenen Hof mit fürstlichen Gewohnheiten zu gestalten (beispielsweise minimale Zahl an Trompetern etc.). Die neuzeitliche Zentralisierung der liechtensteinischen Archivalien ${ }^{104}$ zeigte das Bemühen hinsichtlich einer nachträglichen fachlichen Klassifizierung der Schriftquellen. ${ }^{105}$

Allgemein muss jedoch betont werden, dass Trompeter, mitunter ein Trompeterensemble, einen unerlässlichen Bestandteil des Fürstenhofes bildeten, die Existenz eines Musikensembles (musica) stets eine Angelegenheit der aktuellen Bedürfnisse, Präferenzen und wirtschaftlichen Möglichkeiten des konkreten Fürsten darstellte. ${ }^{106}$

103 Die Theaterfrage behandelt Bohatta, H.: Das Theaterwesen, S. 38-86. Aus Sicht des tschechischen Theaterwesens vgl. die Losung Vaňáč, Martin - Jakubcová, Alena: Alois I. Josef Lichtenštejn. In: Jakubcová, Alena (Hg.): Starší divadlo v českých zemích do konce 18. století. Osobnosti a díla. Praha 2007, S. 343-346; vgl. auch Pavlišová, Jitka: Zámecká divadla rodu Liechtensteinů na českém území. Závěrečná studie z ročního výzkumného projektu (Die Schlosstheater der Familie Liechtenstein auf tschechischem Gebiet. Abschussstudie des Jahresforschungsprojketes). Theatralia, Jg. 13, 2010, Nr. 1: 32-46, Dies.: Ve službách knižete Liechtensteina: několik poznatkủ k provozu zámeckého divadla ve Valticích (In Diensten des Fürsten Liechtenstein: einige Bemerkungen zum Betrieb des Schlosstheaters in Feldsberg). Theatralia, Jg. 18, 2015, Nr. 1, S. 85-105.

104 Stögmann, Arthur: Das Hausarchiv der regierenden Fürsten von und zu Liechtenstein. In: Mitteilungen des österreichischen Staatsarchivs Bd. 56, Wien 2011, S. 503-518. In dieser Studie wird auch die Struktur der liechtensteinischen Schriftgüter in den Archiven der Tschechischen Republik kurz umrissen (insbesondere geht es um die Fonds des Mährischen Landesarchivs).

105 Die Ausgliederung zahlreicher wertvoller Dokumente aus ihrem ursprünglichen Kontext entsprechend des neuzeitlichen Fächerschlüssels erinnert in vielem an die Methoden der destruktiven Archäologie. Singuläre Quellen erscheinen somit im Fürstenarchiv transparenter (einschließlich der editorischen Erarbeitung vieler solcher Quellen durch Herbert Haupt, bei dessen verdienstvoller Tätigkeit freilich eine bestimmte subjektive Auswahl der Quellen deutlich wird), doch erst die konsequente Konfrontierung mit anderen Akten und Rechnungen, die der Autor dieser Studie im Bereich der musikalischen Aktivitäten am Hofe Karls von Liechtenstein vorgenommen hat, vermag die Bedeutung des musikalischen Betriebs und die Stellung der Musiker an den Höfen der einzelnen Fürsten zu klären helfen.

106 Aus Sicht der tschechischen Musikologie wurde bislang ein größerer Teil der Aufmerksamkeit den auf dem Gebiet der Tschechischen Republik lagernden Quellen gewidmet. Relevante Quellen werden heute in Wien aufbewahrt und harren, bis auf einzelne Ausnahmen, noch einer systematischeren Auswertung. Aus Sicht der tschechischen Musikwissenschaft fasste die bisherigen Kenntnisse über den Musikbetrieb am Hofe der Liechtenstein Sehnal, Jiří: Die adeligen Musikkapellen im 17. und 18. Jahrhundert in Mähren. In: Biba, Otto - Wyn Jones, David (Hg.): Studies in Music History. Presented to H. C. Robbins Landon on his 70th Birthday. London 1996, S. 195-217, 266-269, hier S. 215-216, zusammen; vgl. des Weiteren auch Sehnal, Jiří - Vysloužil, Jiř̌i: Dějiny hudby na Moravě (Die Geschichte der Musik in Mähren). Brno 2001, S. 104-105. Einen grundlegenden Beitrag zu der bislang noch nicht flächendeckend erforschten Situation im 18. Jahrhundert publizierte Ágústsson, Jóhannes: Joseph Johann Adam of Liechtenstein, Patron of Vivaldi. Studi Vivaldiani 17, 2017, S. 3-78. In Betracht gezogen werden müssen auch die unwiederbringlichen Verluste von Archivalien am Ende des Zweiten Weltkrieges (insbesondere im Fall der Schriftquellen in 


\section{Zusammenfassung}

\section{... viell fürsten halten ein Musica...}

\section{Hudba a hudebníci na dvoře knížat z Liechtensteina v 17. století}

Takzvaný Politický testament, který někdy kolem roku 1680 sepsal Karel Eusebius kníže z Lichtenštejna pro svého syna a dědice, obsahuje mimo jiné i zásadní, podrobnou pasáž věnovanou hudbě na knížecím dvoře. Hudba zde nefiguruje jen jako jeden z elementů dvorské reprezentace. Tento klíčový aspekt se v textu instrukce setkává s poučeným náhledem na problémy s provozem hudebního souboru i s využitím a charakterem jednotlivých nástrojů. Tématem této studie je především postavení hudebních souborů a hudebníků v rámci struktury aristokratického dvora počínaje Karlem I. (z. 1627) přes dílčí analýzu situace na dvoře jeho syna Karla Eusebia (z. 1686) a konečně s nástinem dosud málo objasněné role hudebníků a hudebního provozu u třetí generace knížat z Lichtenštejna. Určitou kontinuitu lze předpokládat především ve snaze uzpưsobit vlastní dvůr knížecím zvyklostem (například ve věci minimálního počtu trubačů etc.). Obecně je však nutné zdůraznit, že trubači, případně trubačský soubor představovali nezbytnou součást knížecího dvora, existence př́ípadného hudebního souboru (musica) vždy věcí aktuálních potřeb, preferencí a ekonomických možností konkrétního knížete.

Nové Zámky bei Littau), die auch die bislang geringen Kenntnisse über die Existenz von Theatermaterial (Libretti etc.) und Musikalien liechtensteinischer Provenienz einordnen. 
